\title{
A taxonomic review of the crab spider genus Sidymella (Araneae, Thomisidae) in the Neotropics
}

\author{
Miguel Machado ${ }^{1}$, Catherine Guzati ${ }^{1}$, Rafaela Viecelli ${ }^{1}$, Diana Molina-Gómez ${ }^{1}$, \\ Renato Augusto Teixeira ${ }^{1}$ \\ 1 Laboratório de Aracnologia, Faculdade de Biociências, Pontificia Universidade Católica do Rio Grande do Sul (PUCRS), Avenida Ipiranga \\ 6681, Porto Alegre, RS, Brazil \\ http://zoobank.org/1AC7C423-2E9A-42B0-AD01-902985656BE6 \\ Corresponding author: Miguel Machado (machadom.arachno@gmail)
}

Academic editor: Danilo Harms • Received 28 March 2019 • Accepted 2 May 2019 • Published 29 May 2019

\begin{abstract}
Four Neotropical species of Sidymella Strand, 1942, S. furcillata Keyserling, 1880, S. longispina (Mello-Leitão, 1943), S. lucida (Keyserling, 1880), and S. kolpogaster (Lise, 1973) are redescribed from both sexes. The holotype of S. nigripes (Mello-Leitão, 1947) is lost and this taxon is considered a species inquierenda. Sidymella obscura (Mello-Leitão, 1929), S. parallela (Mello-Leitão, 1929), and S. spinifera (Mello-Leitão, 1929) are all nomina dubia. Two new species are described: Sidymella excavata sp. nov. (males and females) and S. marmorata sp. nov. (female).
\end{abstract}

\section{Key Words}

crab spiders, morphology, new records, Stephanopinae, Stephanopis

\section{Introduction}

Crab spiders (Araneae, Thomisidae) are distributed worldwide but the highest diversity is found in tropical regions (WSC 2019). The group has been studied in many recent phylogenetic works, but its relationships are still being discussed (Benjamin et al. 2008; Benjamin 2011; Ramírez 2014; Wheeler et al. 2017) and broader relationships among basal thomisids such as the subfamily Stephanopinae remain weakly supported and unstable (Ramírez 2014). The presence of cheliceral teeth, which was previously considered as a synapomorphy for this group (Ono 1988), was recovered as a plesiomorphy by Benjamin (2011), and this subfamily remains as the most controversial and the least studied group in Thomisidae; it has many genera in need of revision and a considerable number of species yet to be described (Benjamin 2011). Based on the work of Mello-Leitão (1929), subsequent efforts were made to update the taxonomy of some Neotropical stephanopines (Lise 1973, 1981; Bonaldo and
Lise 2001; Machado et al. 2015, 2017; Silva-Moreira and Machado 2016; Prado et al. 2018). However, many genera are still known only from the original descriptions and poor diagnoses, and the accurate identification of many species is practically impossible.

The genus Sidymella Strand, 1942 is a prime example of such difficulties. The genus has a disjunct Gondwanan distribution, with 11 described species occuring in Australia and New Zealand whilst 10 are found in the Neotropics (WSC 2019). Sidymella is currently defined by a convex prosoma, both anterior and posterior eye rows recurved, anterior tibiae and metatarsi (I and II) with stout and spiniform macrosetae, and opisthosoma posteriorly bifurcated (Strand 1942; Mello-Leitão 1929; Lise 1973). Although the Neotropical Sidymella have been revised by Lise (1973), this author focused on somatic characters to describe and diagnose the species, neglecting both external and internal structures of female genitalia and the position, shape, and size of palpal apophyses of males. Therefore, the present paper provides a taxonomic review of the Neo- 
tropical Sidymella, where the males of $S$. furcillata and $S$. longispina are described for the first time, two new species added to the genus, and new synonyms established.

\section{Methods}

The examined specimens belong to the Museo Argentino de Ciencias Naturales "Bernardino Rivadavia", Buenos Aires (MACN, Martín Ramírez), Museu de Ciências e Tecnologia da Pontifícia Universidade Católica do Rio Grande do Sul, Porto Alegre (MCTP, A. A. Lise), Museu de Ciências Naturais da Fundação Zoobotânica do Rio Grande do Sul, Porto Alegre (MCN, R. Ott), and the Museum of Comparative Zoology of Harvard, Cambridge (MCZ, G. Giribet and L. Liebensperger).

The terminology for somatic and copulatory structures follows Machado et al. (2018). Photos of copulatory structures were taken with a Multipurpose Zoom Microscope Leica M205A with a digital camera, and scanning electron microscopy were made with a Philips XL 30 Field Emission ESEM from the Centro de Microscopia e Microanálises (CEMM) of the Pontifícia Universidade Católica do Rio Grande do Sul (PUCRS). All measurements were taken in millimeters. Morphology abbreviations: ALE - anterior lateral eyes, AME - anterior median eyes, MOQ - median ocular quadrangle, PLE-posterior lateral eyes, PMEposterior median eyes, RTA — retrolateral tibial apophysis.

\section{Results}

\section{Sidymella Strand, 1942}

Sidyma Simon, 1895: 1056. Type species: Stephanopis lucida Keyserling, 1880; by original designation and monotypy; Berland 1913: 95, pl. 9, figs 51-56. Preoccupied by Walker (1856) in Lepidoptera. Sidymella Strand, 1942: 399 (generic replacement name).

Diagnosis. Sidymella is similar to Coenypha Simon, 1895 and some species of Stephanopis (e.g. S. antennata, S. ditissima, and $S$. nodosa) and these species share a male palp with a long, thin and curled embolus, well-developed pars pendula, and a retrolateral tibial apophysis with a short basal branch (Figs 1A, B, 11C-F, 13D); the epigynes have a septum formed by the posterior folds of the epigynal plate and long and coiled copulatory ducts (Figs 1C, D, 10C-F). However, Sidymella species can be recognized and distinguished from these genera by having a bifid opisthosoma (Fig. 1E, F), presence of spiniform macrosetae on the mesial surface of femora I and above the ALE (Fig. 10A, B), the epigyne have a single pair of walnut-shaped spermathecae compartmentalized in several smaller chambers, with accessory glandular heads (Figs 4D, F, 6D, F, 8D, F, 10D, F, 12D, F, 13D, F) while the male palp has a tibial trichobothrium and the RTA have a nodose surface (Fig. 1A, B) unlike other Stephanopinae which present a grooved RTA (with parallel creases on its surface).
Description. Small spiders (total length 3.00-3.96 in males, 4.68-7.93 in females) with slight sexual size dimorphism, presenting evidence of predominant green colouration in vivo (Fig. 1E, F). Prosoma longer than wide, covered with short and conical setae. Both anterior and posterior eyes disposed in two recurved rows; ALE almost two times larger than the other eyes; ocular macrosetae may be present only above the ALE or on the MOQ area; sternum heart-shaped with concave anterior border, and clypeus with a pair of macrosetae; labium trapezoidal and endites with rounded edges, longer than wide. Opisthosoma with two projections of different sizes, shapes and angles in relation to the opisthosomal axis, varying from rounded and short ones, disposed horizontally/posteriorly, or long, acute and vertically oriented (Figs 2A-F, 3A-F). Leg formula: 1-2-4-3; anterior tibiae and metatarsi (I and II) ventrally armed with stout macrosetae. Epigynum with membranous and hyaline copulatory ducts, long spermathecae with many chambers or with a single median constriction (Fig. 10D); male palp with RTA rounded or truncated, discoid tegulum and ribbon-like embolus and (Fig. 10C-F).

Composition. Six Neotropical species: Sidymella excavata sp. nov., S. furcillata (Keyserling, 1880), S. longispina (Mello-Leitão, 1943), S. lucida (Keyserling, 1880), S. marmorata sp. nov., and S. kolpogaster (Lise, 1973); 11 additional species from the Australasian region that are not within the scope of the present study.

Distribution. Ecuador, Colombia, Peru, Brazil, Argentina, and Uruguay (Fig. 15).

\section{Sidymella excavata Machado \& Guzati, sp. nov.}

http://zoobank.org/4D59E033-C71C-4588-ADBC-6563B92ACDAE Figures 2D, 3D, 4, 5

Type material. Holotype + , ECUADOR: Cerro Troya, Carchi, 043'59.7"N, 7741'00.3"W, L. Pena leg. (MCZ 133396). Paratypes: §̂, ECUADOR: Pichincha, $0^{\circ} 15^{\prime} 00.0^{\prime \prime} \mathrm{S}, 78^{\circ} 35^{\prime} 00.0^{\prime \prime} \mathrm{W}, 19$. iv.1988, W. Maddison leg. (MCZ 133397); q, COLOMBIA: Cundinamarca, Mosquera (Mondoñedo), 441'0.06"N, 74¹5'0.25"W, 20.x.2000, E. Florez \& J. Pinzon leg. (ICN 3404).

Material examined. COLOMBIA: $2 \mathrm{j}$, Magdalena (Santa Marta), $11^{\circ} 12^{\prime} 54^{\prime \prime} \mathrm{N}, 7^{\circ} 06^{\prime} 01^{\prime \prime W}, 18 . i v .1977, \mathrm{~W}$. Galvis \& A.J. Moreno leg. (ICN-Ar 9140); 1 q 1j, Quindío (Estación Bremen), 440'0"N, 75³9'0"W, 14-20.iv.1998, P. Ariza leg. (MPUJ 45511); 1 9 , 1j, same data as preceding (MPUJ 11490); 1ㅇ, Cundinamarca, Mosquera (via a La Mesa), 440'12.0"N, 74¹6'00.7"W, 20.x.2000, E. Florez \& J. Pinzon leg. (ICN-Ar 3404); 2 , Mosquera (Desierto de Zabrinsky), 444'30"N, 74¹4'8"W, 23.ii.2002, L. Benavides, C. Niño, A. Castañeda \& G. Mora leg. (ICN-Ar 2157); 2ᄋ, 5j, 06.iv.2002, same locality and collectors (ICN-Ar 2146); 4, 7j, same locality, April 2006, 


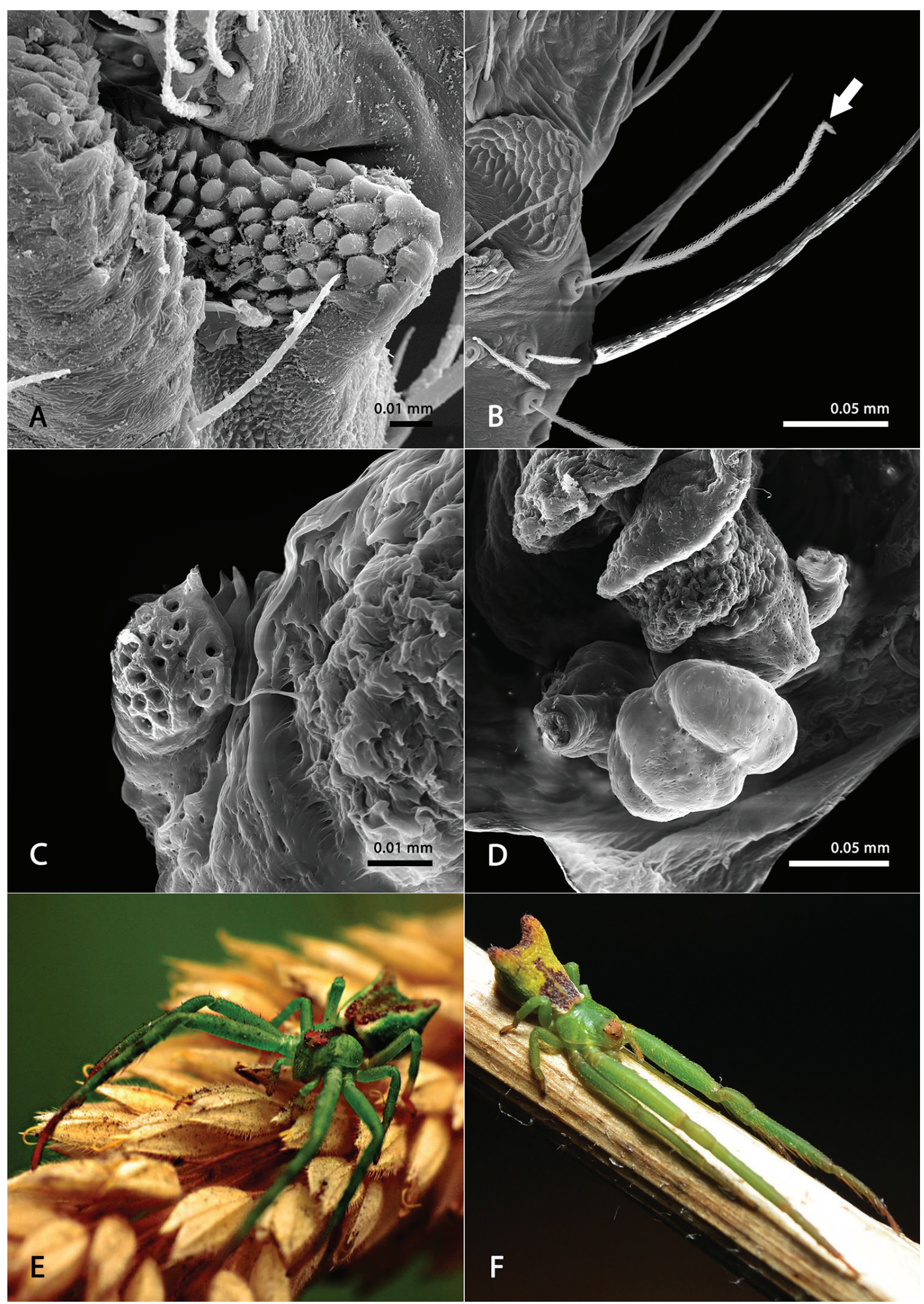

Figure 1. a-d Diagnostic sexual features of Sidymella a Detail of the nodose RTA surface on male palp b Tibial trichobothria on male palp (indicated by an arrow) $\mathbf{c}$ Glandular head on spermatheca $\mathbf{d}$ Walnut-shaped spermatheca $\mathbf{e}, \mathbf{f}$ Photos of live specimens of Sidymella lucida. Photo credits: e Diego Galarraga Sugoniaev; f Damián Hagopián. 


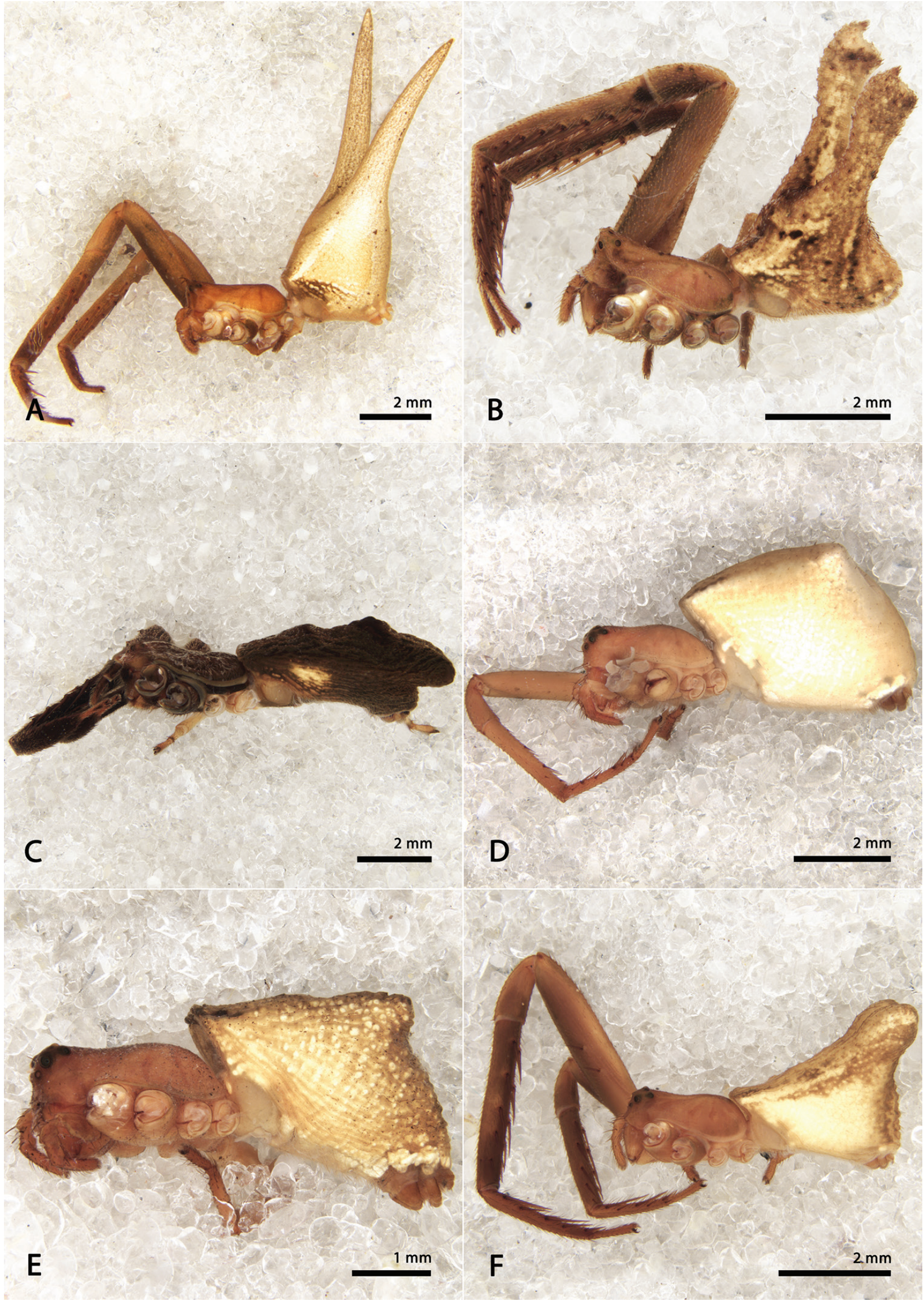

Figure 2. Habitussen of females, lateral a Sidymella longispina b Sidymella furcillata c Sidymella kolpogaster d Sidymella excavata sp. nov. e Sidymella marmorata sp. nov. f Sidymella lucida. 

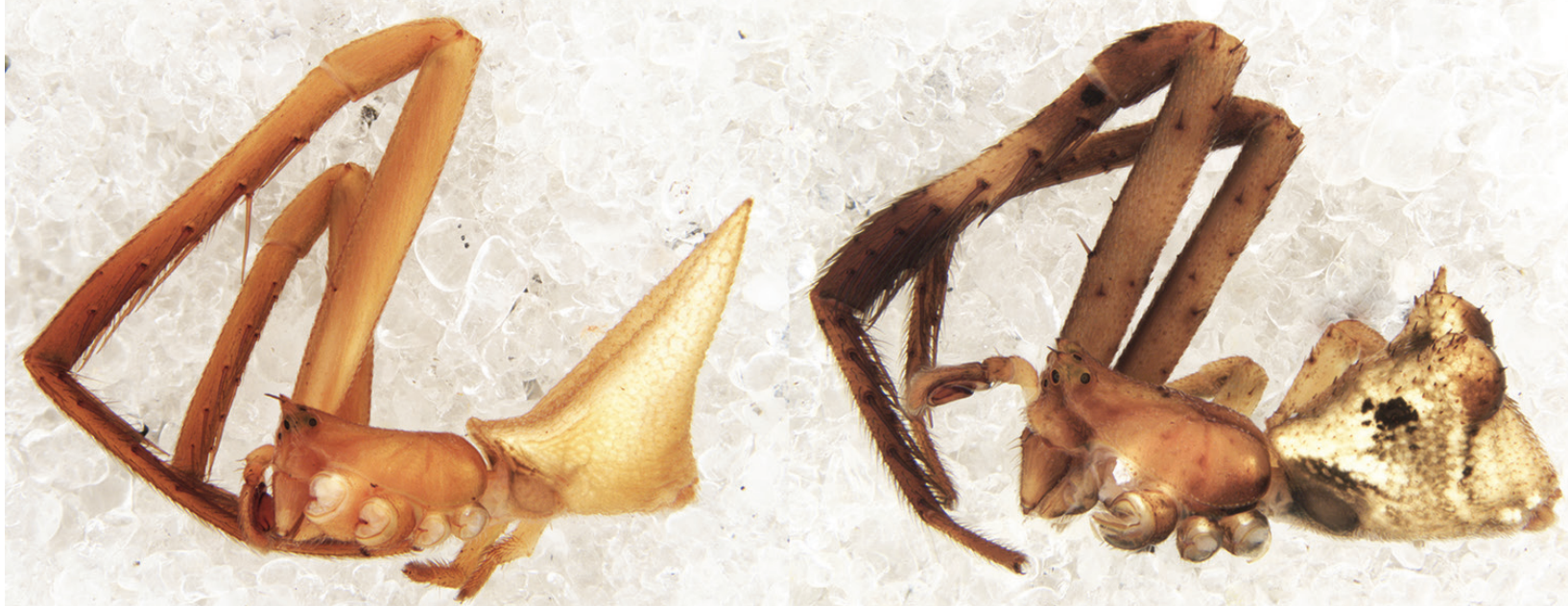

A

$1 \mathrm{~mm}$

B

$1 \mathrm{~mm}$
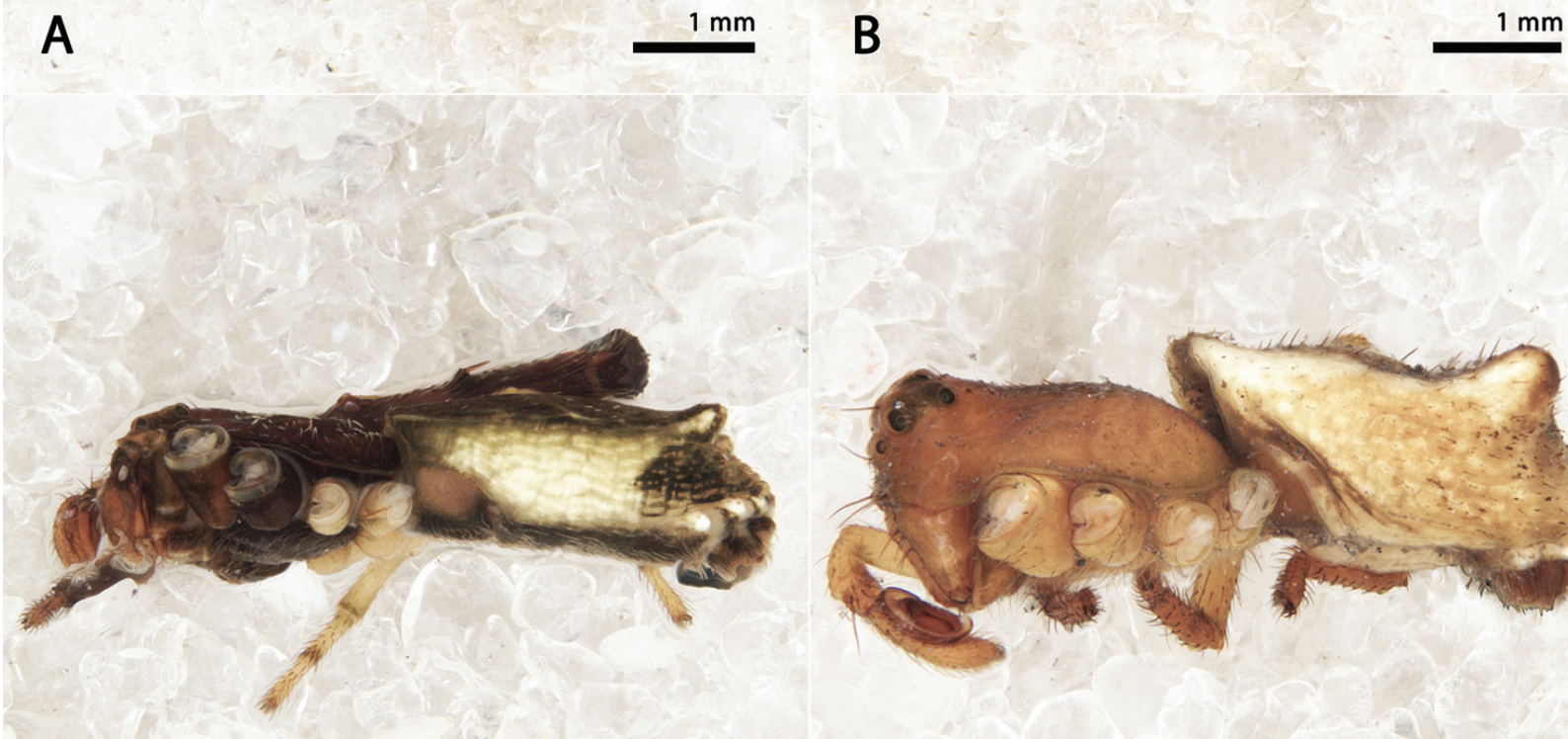

C

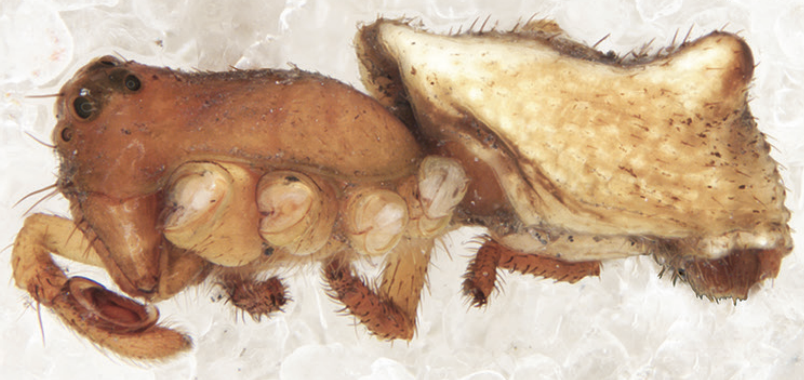

$1 \mathrm{~mm}$ - D
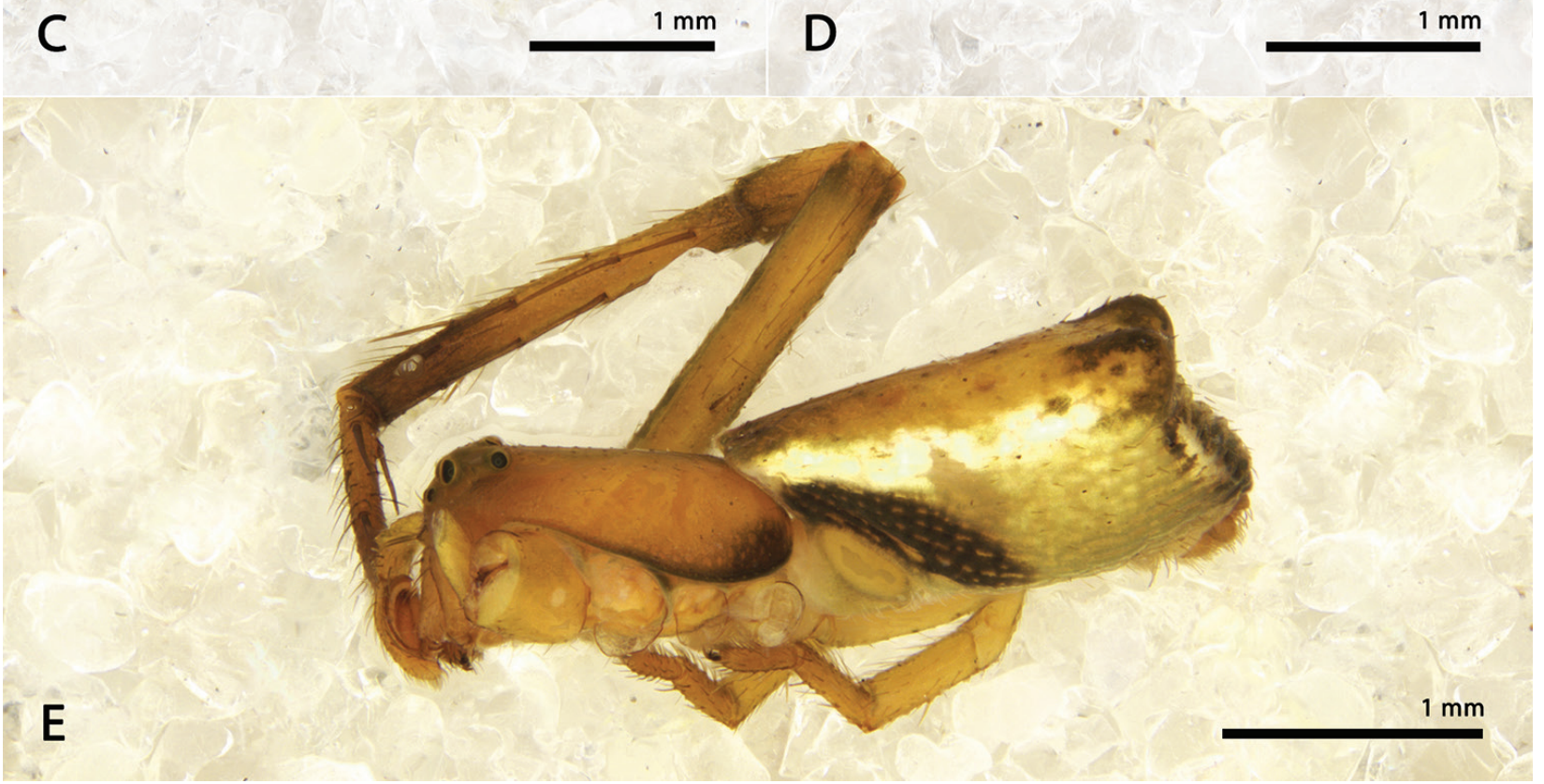

Figure 3. Habitussen of males, lateral a Sidymella longispina b Sidymella furcillata c Sidymella kolpogaster d Sidymella excavata sp. nov. e Sidymella lucida. 

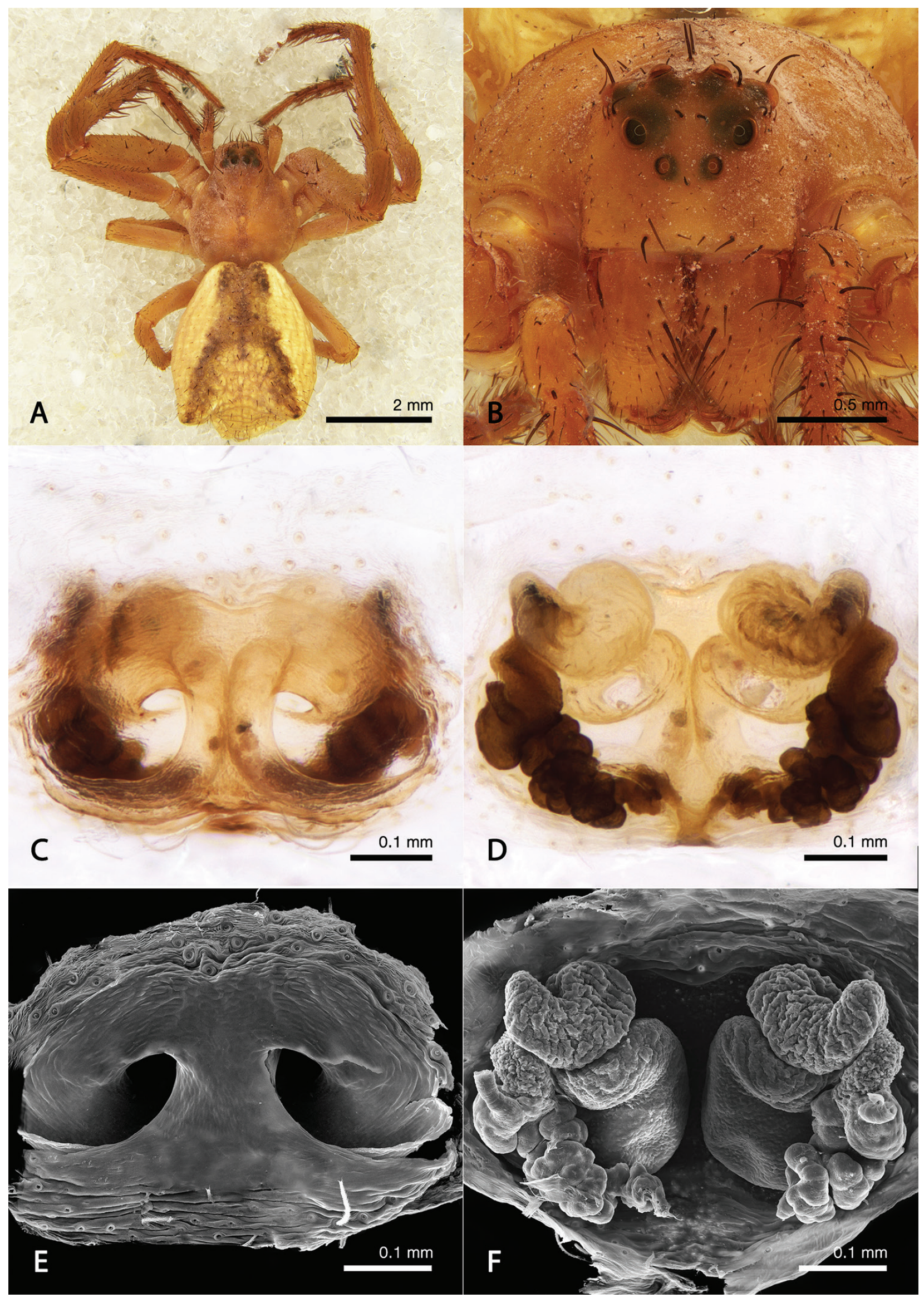

Figure 4. Female of Sidymella excavata sp. nov. a habitus, dorsal b prosoma, anterior c, e epigynum, ventral d, $\mathbf{f}$ epigynum, dorsal. 


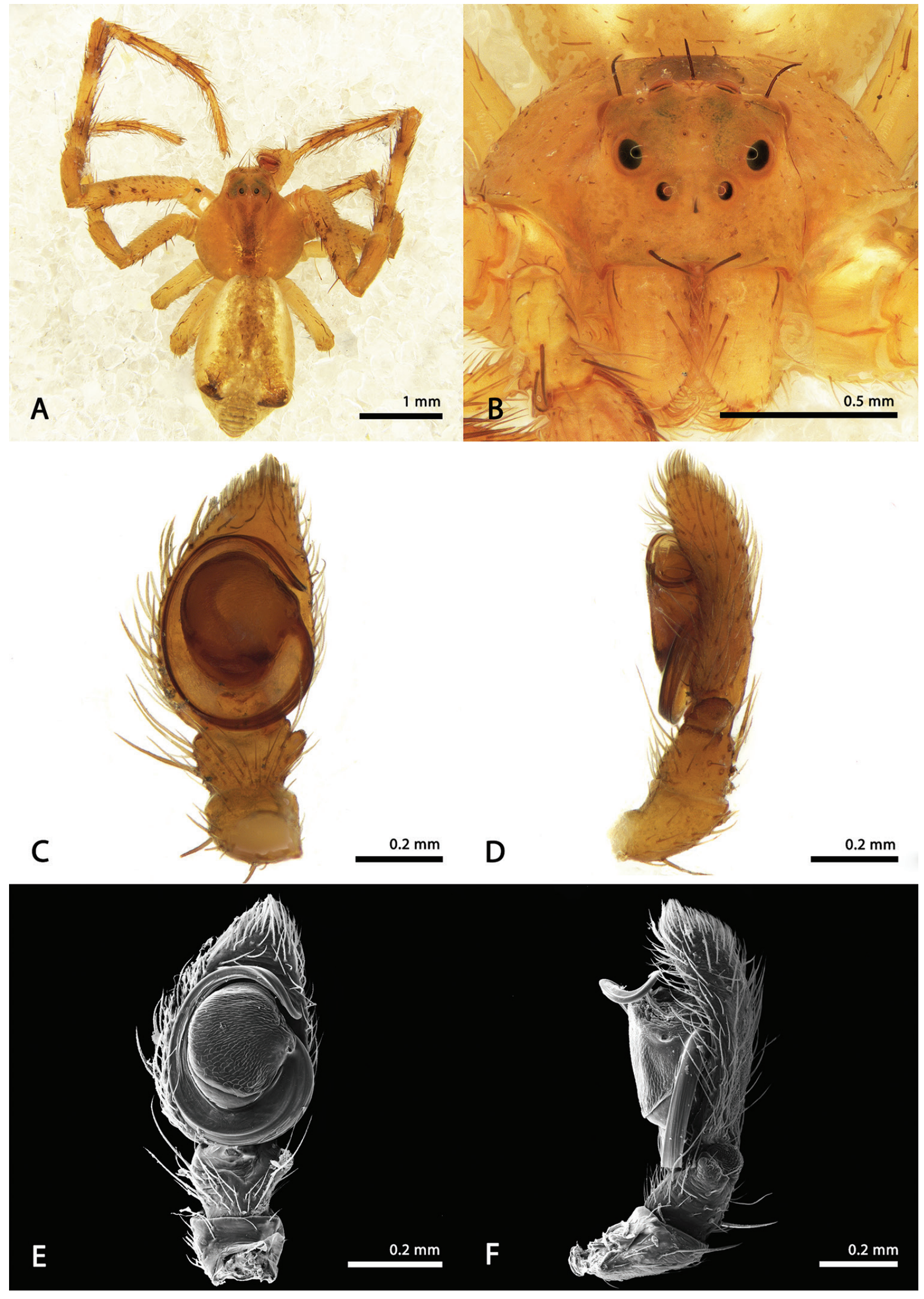

Figure 5. Male of Sidymella excavata sp. nov. a habitus, dorsal b prosoma, anterior c, e left palp, ventral view d, f left palp, retrolateral view. 
J. Martínez leg. (ICN-Ar 10500); 19, 23.ii.2002, same locality, C. Niño, G. Mora, A. Castañeda \& J. Martínez leg. (CAUD-216-ARA 139); 1, 27.vii.2002, same locality, J. Martínez, C. Niño \& G. Mora leg. (CAUD-216-ARA 366); 1 q, iv.2002, same locality, C. Niño, G. Mora, A. Castañeda \& J. Martínez leg. (CAUD-216-ARA 1944); 19 , 08.ix.2016, same locality, D. Molina leg. (ICN-Ar 10449); 1ㅇ, same data as preceding (ICN-Ar 10498). 1 ㅇ, $2 \mathrm{j}$, Boyacá, Villa de Levya, 540'21"N, 73²7'42"W, 09.vi.2001, L. Benavides leg. (ICN-Ar 1248). 1ð̂, Valle del Cauca, Saladito, 33 $34^{\prime} 15.78^{\prime \prime N}, 76^{\circ} 36^{\prime} 9.27^{\prime \prime W}$, W. Eberhard leg. (MCZ 133395). ECUADOR: $1 \jmath^{\lambda}$, Quito, Pichincha, $0^{\circ} 15^{\prime} 00^{\prime \prime} \mathrm{S}, 78^{\circ} 35^{\prime} 0^{\prime \prime} \mathrm{W}, 19 . \mathrm{iv} .1988$, W. Maddison leg. (MCZ 133397). 1ð^, 2j, Azuay, Cerro Tinajillas, $3^{\circ} 10^{\prime} 60^{\prime \prime} \mathrm{S}$, 7902'00"W, 18-21.iii.1965, L. Pena leg. (MCZ 133403).

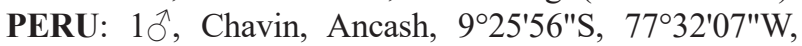
14.i.1972 (MACN-Ar 19068). ARGENTINA: 30̄, 4 j, Catamarca, Las Chacritas, 2701'19.25"S, 6555'24.97"W, 20.i.1987, P. Goloboff leg. (MACN-Ar 19098).

Etymology. The specific name refers to the shape of the anterior border of the opisthosoma with a remarkable median re-entrance/excavation.

Diagnosis. Females of $S$. excavata sp. nov. resemble those of $S$. marmorata sp. nov. by the large body size, short opisthosomal projections and long copulatory ducts; however, they can be distinguished by the presence of long needle-shaped macrosetae on the ocular area (Fig. 4B), and by having five mesial macrosetae on femora I and II (Fig. 4A). Males are similar to those of S. luci$d a$, but can be recognized by cephalic setae and a basal branch of the RTA which is truncated instead of conical (Fig. 5C-F).

Description. Female: Prosoma, sternum and legs totally orange; legs I and II covered by numerous spiniform setae; opisthosoma predominantly light-yellow with a dark median stain on the dorsum that splits posteriorly towards to the tips of the projections (Fig. 4A). Epigynum with a wide septum formed by the median junction of the posterior folds of the epigynal plate (Fig. 4C, E); copulatory ducts hyaline, long and coiled, leading to a pair of walnut-shaped spermathecae (Fig. 4D, F).

Measurements: eyes sizes and interdistances: AME 0.03, ALE 0.09, PME 0.05, PLE 0.03, AME-AME 0.15, AME-ALE 0.09, PME-PME 0.17, PME-PLE 0.17 , MOQ length 0.41 , MOQ width 0.17 ; leg formula: 1-2-4-3: leg I - femur 3.40/ patella $1.36 /$ tibiae 3.04/ metatarsus $2.04 /$ tarsus $0.88 /$ total 10.72 ; II $-2.68 / 1.12 /$ 2.08/ 1.72/ 0.68/ 8.28; III - 1.40/ 0.72/ 0.88/ 0.80/ 0.60/ 4.44; IV - 1.76/ 0.76/ 1.12/ 0.96/ 0.60/ 5.20. Total body length 5.48; prosoma 2.48 length, 2.04 wide; opisthosoma length 3.00; clypeus 0.35 height; sternum 1.12 length, 1.04 width; endites 0.56 length, 0.20 width; labium 0.32 length, 0.36 width.

Male: Prosoma yellow with a darker median stain; legs I and II yellow with brownish spots on the femo- ral setiferous tubercles and at the distal portion of each leg segment; legs III and IV light-yellow. Opisthosoma as in female (Fig. 5A). Palpi with a well-developed pars pendula and embolus emerging at three o'clock, curling at the tip (Fig. 5C-F); basal branch of RTA stout and directed retro laterally while the RTA is short, rounded and oriented vertically (Fig. 5D, F).

Measurements: eyes diameters and eyes interdistances: AME 0.05, ALE 0.07, PME 0.05, PLE 0.05, AME-AME 0.13, AME-ALE 0.05, PME-PME 0.11, PME-PLE 0.13 , MOQ length 0.17 , MOQ width 0.11 ; leg formula: 1-2-4-3: leg I - femur 2.04/ patella $0.72 /$ tibiae $1.92 /$ metatarsus $1.68 /$ tarsus $0.76 /$ total 7.12 ; II $-1.44 / 0.60 /$ $1.20 / 1.12 / 0.60 / 4.96$; III $-0.72 / 0.60 / 0.56 / 0.40 / 0.40 /$ 2.68 ; IV $-0.96 / 0.36 / 0.60 / 0.52 / 0.40 / 2.84$. Total body length 3.12; prosoma 1.56 length, 1.24 wide; opisthosoma length 1.56 ; clypeus 0.19 height; sternum 0.68 length, 0.62 width; endites 0.35 length, 0.13 width; labium 0.17 length, 0.25 width.

Distribution. COLOMBIA: Magdalena, Quindío, Cundinamarca, Boyacá, and Valle del Cauca; ECUADOR: Pichincha and Azuay; PERU: Chavin; ARGENTINA: Catamarca (Fig. 15).

\section{Sidymella furcillata (Mello-Leitão, 1944)}

Figures 2B, 3B, 6, 7

Sidyma spinifera Mello-Leitão, 1943: 209, f. 36 (q; preoccupied by Mello-Leitão 1929).

Sidyma multispinulosa Mello-Leitão, 1944: 4 (replacement name). Lise 1973: 13, figs 24-33 (holotype $q$ from Santa Catarina, Brazil, P. Buck leg., MNRJ 41934, examined). (New synonymy)

Stephanopis furcillata Keyserling, 1880: 179, pl. 4, fig. 98 (holotype from Santa Cruz, Rio Grande do Sul, Brazil, Hensel leg., ZMB 2406, examined). (New combination)

Material examined. ARGENTINA: 1j, Misiones, San Vicente, 26 55'12"S, 54'31'12"W, 12.i.2005, L. Lopardo et al. leg. (ICN-Ar 27642); 1j, same locality, viii.1954, Schiapelli de Carlo leg. (ICN-Ar 19099); 2j, same locality, 1954, Schiapelli de Carlo leg. (ICN-Ar 19096). BRAZIL: $1 \delta^{\lambda}$, Bahia: Maracás, $13^{\circ} 28^{\prime} 15^{\prime \prime S}$, 40²6'16"W, 14.iii.2012, E.S. Araújo \& A.S Medeiros leg. (UFMG 15165); 1ठ̃, São Paulo, Botucatu (Rubião Júmior), 2253'53"S, 48²9'23"W, 15.ii.1966, V.C. Jesus leg. (MNRJ 10391); 1옹 Paraná: Colombo, $25^{\circ} 17^{\prime} 31^{\prime \prime S}, 49^{\circ} 13^{\prime} 26^{\prime \prime} \mathrm{W}, 02 . x i i .1990$ (MCN 20684); $10^{\top}$, Três barras do Paraná, 2525'08"S, 53¹0'51"W, 20-26.ii.1993 (MCN 23043); 19, Curitiba (Parque Birigui), 2525'31.58"S, 49¹8'38.69"W, 01.xii.1990, A.B. Bonaldo leg. (MCN 20626); 1 을 Curitiba, $25^{\circ} 25^{\prime} 47^{\prime \prime S}$, 49¹6'19"W, 15.iv.2005, J. Ricetti leg. (MCTP 37237); 1 , , Santa Catarina: Itapiranga, $27^{\circ} 10^{\prime} 08^{\prime \prime S}, 53^{\circ} 42^{\prime} 43^{\prime \prime} \mathrm{W}$, Pio Buck leg. (MNRJ 41934); 2 q, 3j, Rancho Queimado, 2740'22"S, 4901'19"W, 13-15.i.1995 (MCN 26464);

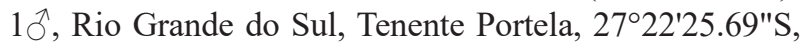
534'32.43"W, 11.ix.1976, S. Scherer leg. (MCN 4862); 
19 , Nonoai (Parque Estadual de Nonoai), 27²7'21.25"S, $53^{\circ} 6^{\prime} 46.98^{\prime \prime} \mathrm{W}, 14.1 .1985$, A.A. Lise leg. (MCN 13077); 1 , , Arroio do Meio, 29²3'47.15"S, 5156'54.26"W, 09.i.1985, same collector (MCN 13071); 1j, Iraí, $27^{\circ} 11^{\prime} 38^{\prime \prime S}, 53^{\circ} 15^{\prime} 03^{\prime \prime} \mathrm{W}, 20$ September 1975, same collector (MCN 8084); $10^{\Uparrow}$, Derrubadas (Parque Estadual do

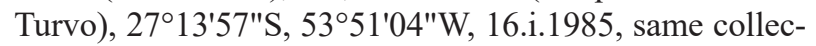
tor (MCN 13057); 1ㅇ, same locality, 01 February 1996, A.B. Bonaldo, A. Kury \& R. Pinto-da-Rocha leg. (MCN 27100); 1j, 27-31.viii.2003, R. Ott leg. (MCN 37827); 1j, Caxias do Sul, 2907'17"S, 5101'07"W, 18-21.xi.1993

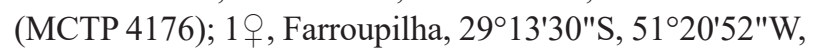
29.ix.1978, H. Bischoff leg. (MCN 8268); 1 q, 2 j, same data as preceding (MCN 8270); 10, Estrela Velha (Barragem de Itaúba), 29¹5'19"S, 5313'36"W, 07.iii. 2001, R. Ott leg. (MCN 33700); 1 q, São Francisco de Paula (Barragem Passo do Inferno), 29¹6'29"S, 5044'15"W, 26.ix.2000, M.A.L. Marques leg. (MCN 33220); 1ㅇ, São Francisco de Paula, 29²7'00"S, 50³4'59"W, 10.xii. 2007, L. Moura \& R. Moraes leg. (MCN 43748); 1 \%, Parobé (Rio dos Sinos), 29³7'29"S, 5049'56"W, 11.v. 2008, E.N.L. Rodrigues leg. (MCN 47943), 1 q, Maquiné, 29³9'41"S, 50¹2'47"W, 08-09.iii.1998, L.A. Moura leg. (MCN 29060); 1, Campo Bom, 2940'44"S, 51³'10"W, 19-20.v.1986, C.J. Becker leg. (MCN 15080); 2 , , Santa Maria, 2940'59"S, 5348'00"W, 17.iv.1996, C.B. Kotzian \& L. Indrusiak leg. (MCTP 40093); 1j, Montenegro, 2941'20"S, 5127'39"W, 06.viii.1977, H. Bischoff leg. (MCN 6822); 1j, same locality, 03.xi.1977, A.A. Lise leg. (MCN 7141); 1§̄, 1, 3j, same locality, 15.xii.1977, same collector (MCN 7510); 19, same locality, 20.xii.1977, same collector (MCN 7614); 1j, same locality, 03.xi.1977, same collector (MCN 8089); 2j, same locality, 29.ix.1977, same collector (MCN 9058); 5j, same data as preceding (MCN 9059); 1오, São Leopoldo, 2945'36"S, 518'49"W, 12.vi.1992 (MCN 24755); 29, 3j, Montenegro (Pesqueiro), 2946'16"S, 51²3'57"W, 12.v.1977, A.A. Lise leg. (MCN 5323); 19, Santo Antônio da Patrulha, 2949'4"S, 50³1'12"W, 18.vii.2000, same collector (MCN 33116); $2 q$, Triunfo (Parque Copesul de Proteção Ambiental), 2951'57"S, 51²1'54"W, 25.i.1990, A.B. Bonaldo leg. (MCN 19344); 1ㅇ, same locality, 13.i.1994, A.F. Franceschini leg. (MCN 24771); 1j, same locality, 25.xi.1994, L.A. Moura leg. (MCN 26116); 1ð̄, 2 , , same locality, 08.i.1997, E.H. Buckup leg. (MCN 28176); 2ㅇ, 1415.i.1997, L.A. Moura leg. (MCN 28263); 3 , , same locality, 16-17.iii.1998, L.A. Moura leg. (MCN 29238); 19, same locality, 05.ii.2003, R. Ott leg. (MCN 35125); 2q, same locality, 29-30.iv.2003, R. Ott leg. (MCN 35740); 2ᄋ, same locality, 06.i.2005, R. Ott leg. (MCN 38352); 1ㅇ, same locality, 01.iii.2005, R. Ott leg. (MCN 39924); 1ㅇ, same locality, 28.v. 2007, E.N.L. Rodrigues leg. (MCN 43383); 1ð̄, 1 ㅇ, same locality, 14.ii.2008, R. Moraes leg. (MCN 44009); 1 , , same locality, 03.iv.2008, A. Barcellos leg. (MCN 44127); 1q, same locality, 26.ii.2010, M.C. Pairet Jr. leg. (MCN 47032); 1 , , same locality, 26.ii.2010, M.A.L. Marques leg. (MCN 47049); 3 , Canoas, $29^{\circ} 55^{\prime} 12^{\prime \prime S}, 51^{\circ} 10^{\prime} 48^{\prime \prime W}$, 13.xii.1990 (MCN
20142); 1ð̄, 1ㅇ, 2j, same locality, 24.i.1991, H. Galileo \& E.H. Buckup leg. (MCN 20431); 1j, same locality, 25.xii.1983, A.D. Brescovit leg. (MCN 11904); 3 q Triunfo, 2956'34"S, 5143'4"W, 20.x.1977, H. Bischoff leg. (MCN 6900); 3 q, same locality, 28.xi.1977, same collector (MCN 7314); 19 , Triunfo (Parque Estadual Delta do Jacuí), 2957'16"S, 51²1'55"W, 23.ii.1999, A.B. Bonaldo leg. (MCN 30488); 1j, Porto Alegre, 3001'58"S, 51ํ'13'48"W, 04.ix.1977, A.A. Lise leg. (MCN 6469); 1ㅇ, same locality, 17.xii.1983, A.D. Brescovit leg. (MCN 11882); 2ิ̂, 19, same locality, 18.i.1992, A.D. Brescovit leg. (MCN 21958); 1 q, same locality, 2010, biology students leg. (MCTP 41329); 1j, Porto Alegre (Morro Santana), 3002'34"S, 51 ${ }^{\circ} 08^{\prime} 39^{\prime \prime W}$, 22.x.1981, A.A. Lise leg. (MCN 11421); 1j, Viamão (Estação Experimental Fitotécnica Águas Belas), 3002'51"S, 5100'53"W, 24.i.1977, A.A. Lise leg. (MCN 5140); 1ठ, same locality, 06.i.1977, same collector (MCN 5763); 1 + , Viamão (Escola Marista), 3004'41"S, 5103'02"W, 1994, A. Braul leg. (MCTP 4726); 3j, Porto Alegre (Ponta Grossa), 30¹0'29"S, 51¹1'50"W, 03.ix.1975, A.A. Lise leg. (MCN 3029); 1j, same locality, 07.v.1976, same collector (MCN 8086); 3j, Viamão (Morro do Coco), 30¹6'11"S, 51ํํ'15"W, 04.x.1975, A.A. Lise leg. (MCN 8085); 1 , same locality, 25.vii.1985, same collector (MCN 13378); 1j, Viamão, 304'51"S, 51¹'22"W, 12.i.1996, same collector (MCTP 8365); 19, same locality, 23.i.1996, same collector (MCTP 9294); 3j, same locality, 02.xii.1994, A.A. Lise \& A. Braul leg. (MCTP 5876); 5j, same locality, 12.vii.1994, A.A. Lise leg. (MCTP 5244), 1, 1j, Guaíba, 306'50"S, 51¹9'30"W, 09.i.1996, A.A. Lise leg.

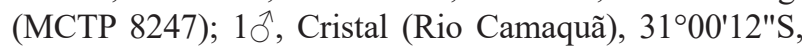
$52^{\circ} 04^{\prime} 02^{\prime \prime W}$, 03.ii.2008, E.N.L. Rodrigues leg. (MCN 48665).

Diagnosis. Females of $S$. furcillata are similar to those of S. longispina by having macrosetae above the ALE and long opisthosomal projections that are directed vertically (Fig. 2B); however, in S. furcillata the projections are rounded at the apex with a smaller terminal tubercle (Fig. 2B). They can be also distinguished by having a group of clavate setae on the median region of the prosoma (Fig. $6 \mathrm{~B})$, a triad of mesial macrosetae on femora I, presence of seven pairs of ventral macrosetae on tibiae I, a dark transversal line on the dorsum of opisthosoma (Fig. 6A), and by the long and coiled copulatory ducts (Fig. 6D, F). Males can be distinguished by having tubercles at the apexes of the opisthosomal projections (Fig. 3B), wide pars pendula (Fig. 7C, E), retrolateral tegular process, and the bifid basal branch of the RTA (Fig. 7D, F).

Description. Female: Prosoma dark-yellow to lightbrown, covered by hyaline setae; legs of the same colour as prosoma, with some sparse darker circular taints randomly distributed; dorsal surface of coxae I and II with wide guanine spots (Fig. 6A). Opisthosoma dark-yellow with two transversal dark lines, being the posterior line disposed along the projections (Fig. 6A). 

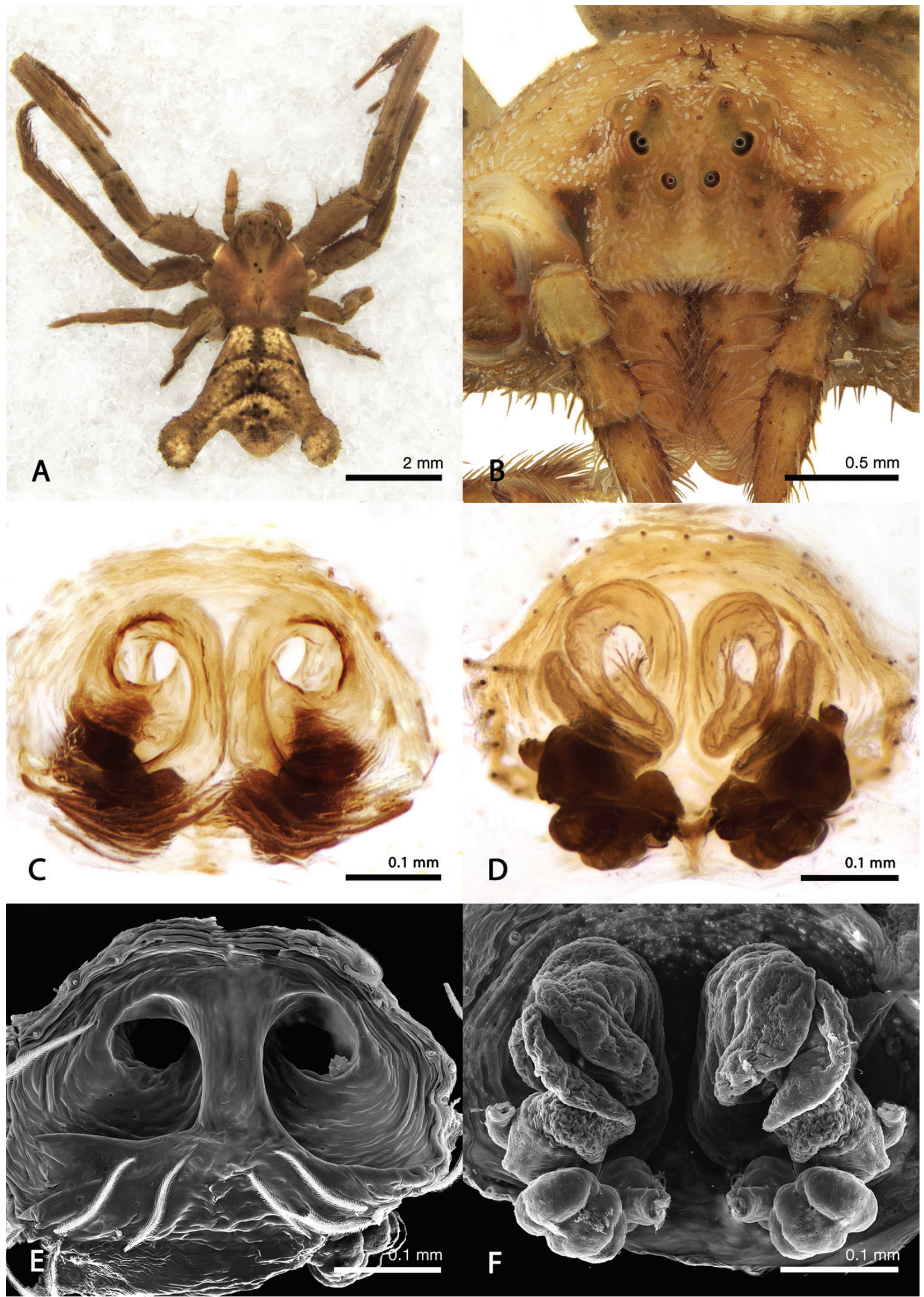

Figure 6. Female of Sidymella furcillata a habitus, dorsal b prosoma, anterior c, e epigynum, ventral d, f epigynum, dorsal. 

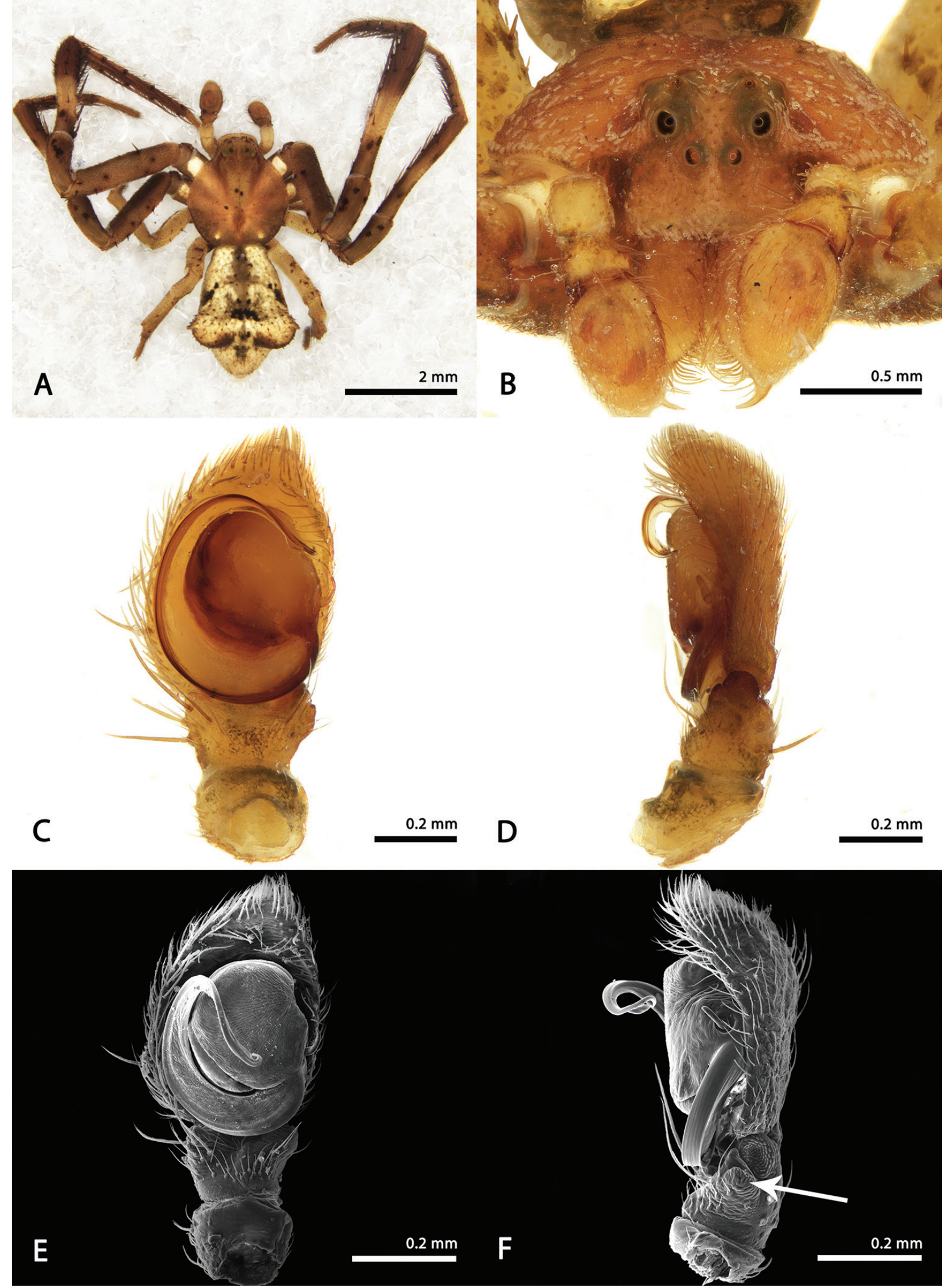

Figure 7. Male of Sidymella furcillata $\mathbf{a}$ habitus, dorsal b prosoma, anterior c, e left palp, ventral view d, f left palp, retrolateral view (white arrow indicates the basal branch of the RTA). 
Measurements: eyes diameters and eyes interdistances: AME 0.05, ALE 0.09, PME 0.05, PLE 0.05, AME-AME 0.13, AME-ALE 0.11, PME-PME 0.15, PME-PLE 0.15, MOQ length 0.33 , MOQ width 0.15 ; leg formula: $1-2-4$ 3 : leg I - femur 3.36/ patella 1.40/ tibiae 3.12/ metatarsus 2.24/ tarsus 0.92/ total 11.04; II - 3.17/ 1.32/ 2.40/ 1.96/ $0.76 / 9.61$; III - 1.16/ 0.68/ 0.92/ 0.60/ 0.52/ 3.88; IV $1.36 / 0.72 / 0.84 / 0.52 / 0.48 / 3.92$. Total body length 5.05 ; prosoma 2.04 length, 2.08 wide; opisthosoma length 3.01 (considering the projections); clypeus 0.29 height; sternum 1.03 length, 0.94 width; endites 0.47 length, 0.29 width; labium 0.27 length, 0.27 width.

Male: Prosoma dark-yellow, legs I and II predominantly light-brown with dark spots; tibiae I yellowish on the median region; legs III and IV yellowish (Fig. 7A). Opisthosoma yellowish with a pair of dorsolateral and a median posterior black spot (Fig. 7A). Embolus long, emerging from tegulum at four o'clock, with wide pars pendula and curled at the tip; RTA rounded and with central excavation (Fig. 7C-F).

Measurements: eyes diameters and eyes interdistances: AME 0.03, ALE 0.07, PME 0.05, PLE 0.05, AME-AME 0.11, AME-ALE 0.09, PME-PME 0.17, PME-PLE 0.13, MOQ length 0.31 , MOQ width 0.11 ; leg formula: 1-2-43: leg I - femur 3.08/ patella 1.32 / tibiae $2.88 /$ metatarsus $2.32 /$ tarsus $0.88 /$ total 10.48 ; II $-2.48 / 1.00 / 2.04 / 1.96 /$ $0.80 / 8.28$; III $-0.76 / 0.52 / 0.76 / 0.56 / 0.48 / 3.08$; IV $1.24 / 0.44 / 0.76 / 0.48 / 0.48 / 3.40$. Total body length 3.96; prosoma 1.96 length, 1.88 wide; opisthosoma length 2.00 (considering the projections); clypeus 0.29 height; sternum 0.90 length, 0.82 width; endites 0.43 length, 0.25 width; labium 0.23 length, 0.27 width.

Distribution. ARGENTINA: Missiones; BRAZIL: Bahia, São Paulo, Paraná, Santa Catarina, and Rio Grande do Sul (Fig. 16).

\author{
Sidymella kolpogaster Lise, 1973 \\ Figures 2C, 3C, 8, 9 \\ Sidyma kolpogaster Lise, 1973: 5, figs 5-9 (†); Lise 1981: 130, figs \\ $1-12(0,+9)$. \\ Sidymella kolpogaster: Brignoli, 1983: 605.
}

Type material. Holotype ${ }_{+}$, BRAZIL: Paraná, Rio Negro, Franciscanos leg. (MNRJ 58065, examined; lost in the fire of the Museu Nacional do Rio de Janeiro). Neotype 9 , BRAZIL: Paraná, Curitiba (Parque Barigui), 2525'12"S, 49¹8'21"W, A.B. Bonaldo leg., 01.xii.1990 (MCN 20622, designated here).

Other material examined. BRAZIL: 19 , Paraná, Rio Negro, 2605'11"S, 4946'47"W (MNRJ 58065); 10َ, San-

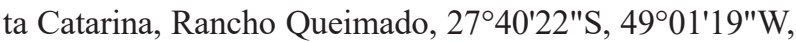
09-13.x.1995, A.A. Lise, A. Braul \& M. Silveira leg. (MCTP 7009); 1요 Rio Grande do Sul, Estrela Velha, $29^{\circ} 15^{\prime} 19^{\prime \prime S}, 53^{\circ} 13^{\prime} 36^{\prime \prime W}$, 21.x.1998, A.B. Bonaldo leg. (MCN 30791); 1j, Arroio do Tigre (Itaúba), 29¹6'47"S,
5306'34"W, 18.iv.1978, A.A. Lise leg. (MCN 8047); 1j, Arroio do Tigre, 29 $19^{\prime} 58^{\prime \prime S}, 5^{\circ} 05^{\prime} 34^{\prime \prime W}$, 23.iv. 1978 (MCN 8150); 19, São Francisco de Paula, 29²4'52"S, 50¹5'24"W, 20.ix.1998, A.A. Lise leg. (MCTP 14446); 1j, same locality, 21-24.iii.1995, same collector (MCTP 12010); 1j, Montenegro, 2941'20"S, 51²7'39"W, 20.xii.1977, A.A. Lise leg. (MCN 7613); 1ð̄, 1 q , Glorinha (Rincão São João), 2949'00"S, 5050'00"W, 14.vii.2000, A.B. Bonaldo leg. (MCN 33053); 1ठ̂, Triunfo (Parque Copesul de Proteção Ambiental), 2951'57"S, 51²1'54"W, 01.ix.2008, E.N.L. Rodrigues leg. (MCN 45434); 1웅, Triunfo (Parque Braskem), 2951'57"S, 51²1'54"W, 04.xii.2009, E.N.L. Rodrigues leg. (MCN 46847); 1j, Porto Alegre (Morro Santana), 3002'34"S, 51 ${ }^{\circ} 08^{\prime} 39^{\prime \prime} \mathrm{W}$, 17.v.1980, A.A. Lise leg. (MCN 9065); 1j, Viamão (Estação Experimental Fitotécnica Águas Belas), 3002'51"S, $51^{\circ} 00^{\prime} 53^{\prime \prime W}$, 12.viii.1994, A.A. Lise leg. (МСTP 5243); 1ㅇ, same locality, 23.ix.1994, same collector (MCTP 5498); 1 đ̄, same locality, 06.v.1994, A.A. Lise \& A. Braul leg. (MCTP 4683); 1 , same locality, 17.i.1977, A.A. Lise leg. (MCN 4995); 1 q, Viamão, 3004'51"S, 5101'22"W, 14.v.2005, R. Jalisco leg. (MCTP 19737).

Diagnosis. Females of $S$. kolpogaster are similar to those of $S$. lucida by the general shape of the spermathecae, short copulatory ducts, and femora I with two mesial macrosetae. However, females of $S$. kolpogaster can be easily distinguished from congeners by curved tibiae I and II, flattened prosoma, dark body colouration, varying from dark-brown to black and contrasting with their vivid yellow legs III and IV (Fig. 8A). The males can also be recognized by their body colour pattern, which is the same as in females, and by their truncated RTA fused with basal branch (Fig. 9D, F).

Description. Female: Prosoma dark-brown, lighter on the ocular area and clypeus, covered by hyaline setae and dorsoventrally compressed (Fig. 8B). legs I and II entirely darkbrown, except for the reduced and yellowish tarsi; metatarsi I and II curved; legs III and IV entirely light-yellow (Fig. 8A). Opisthosoma dark-brown with posterior projections stout and conical. Epigynal plate wide and with short septum; copulatory ducts short and hyaline (Fig. 8C-F).

Measurements: eyes diameters and eyes interdistances: AME 0.07, ALE 0.11, PME 0.05, PLE 0.05, AME-AME 0.17, AME-ALE 0.09, PME-PME 0.23, PME-PLE 0.19, MOQ length 0.21, MOQ width 0.15; leg formula: 1-2-4-3: leg I - femur 4.95/ patella 1.80/ tibiae 4.15/ metatarsus 2.95/ tarsus $0.95 /$ total $14.80 ; \mathrm{II}-4.45 / 1.75 / 3.20 / 2.65 / 0.85 /$ 12.90; III - 1.65/ 0.75/ 1.15/ 1.05/ 0.55/ 5.15; IV - 2.05/ $0.75 / 1.25 / 1.15 / 0.55 / 5.75$. Total body length 7.65 ; prosoma 2.80 length, 2.85 wide; opisthosoma length 4.85 ; clypeus 0.19 height; sternum 1.21 length, 1.20 width; endites 0.68 length, 0.37 width; labium 0.41 length, 0.50 width.

Male: Prosoma and legs colouration as in female (Fig. 9A, B). Palpi with flattened cymbium and dorsal trichobohtria on tibiae; tegulum discoid and embolus thin, slightly curved at the tip and emerging from tegulum at four o'clock (Fig. 9C-F). 

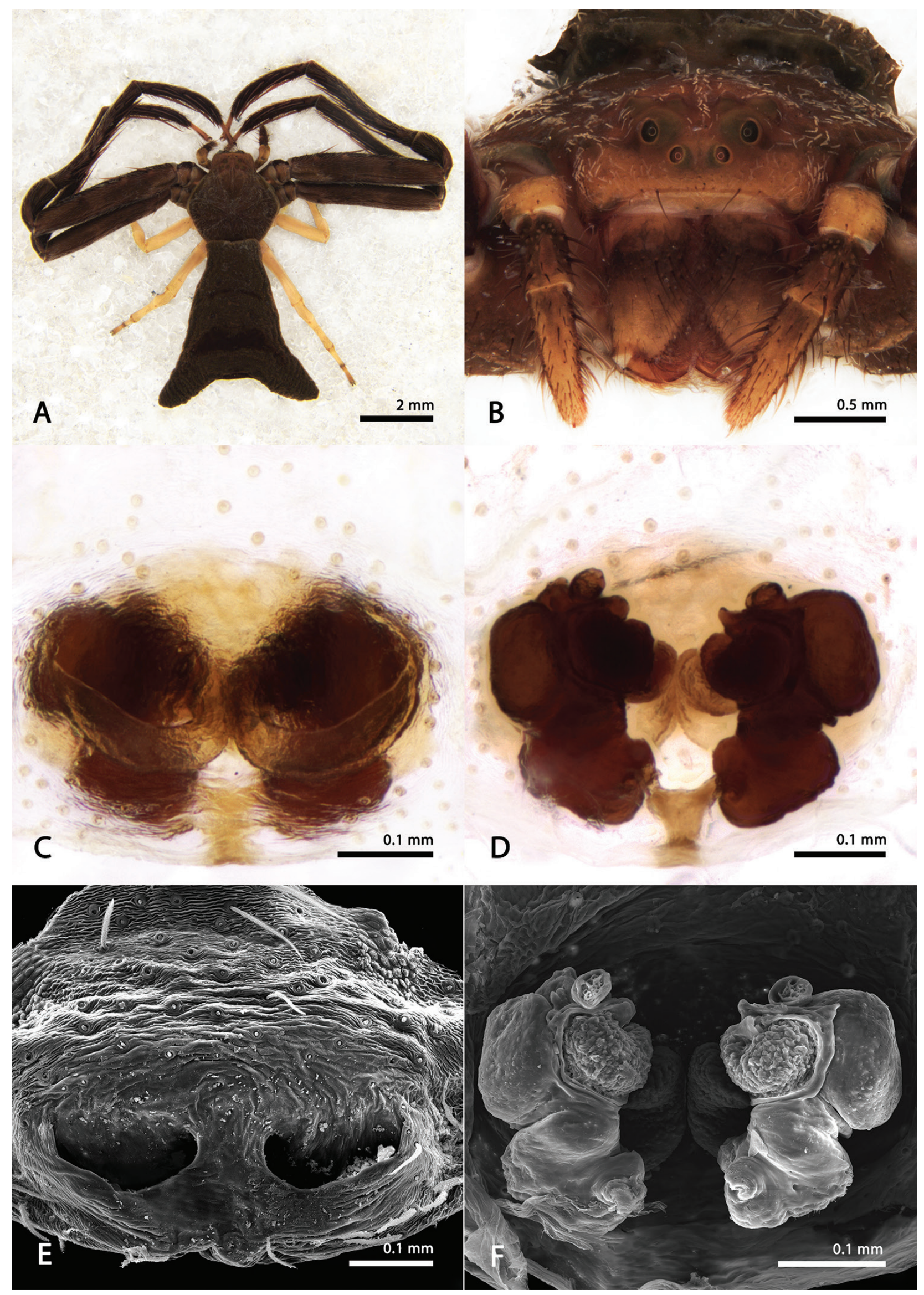

Figure 8. Female of Sidymella kolpogaster a habitus, dorsal b prosoma, anterior c, e epigynum, ventral d, f epigynum, dorsal. 


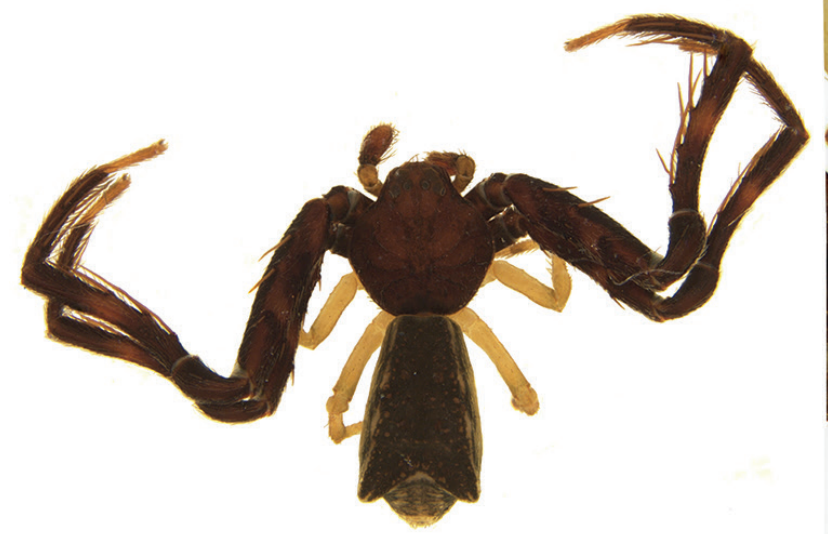

A

$1 \mathrm{~mm}$
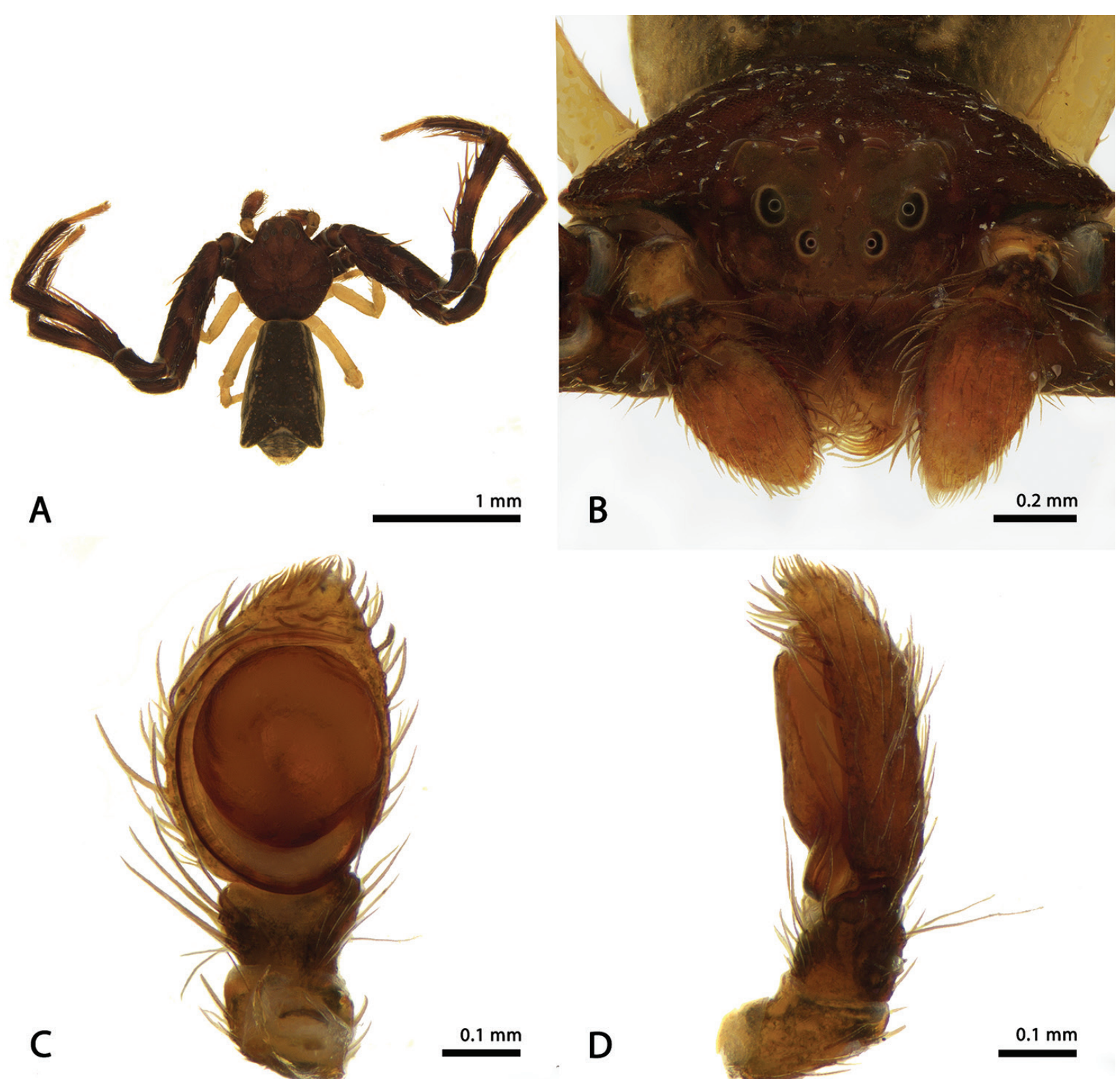

$\underline{0.1 \mathrm{~mm}} \quad \mathrm{D}$

$0.1 \mathrm{~mm}$

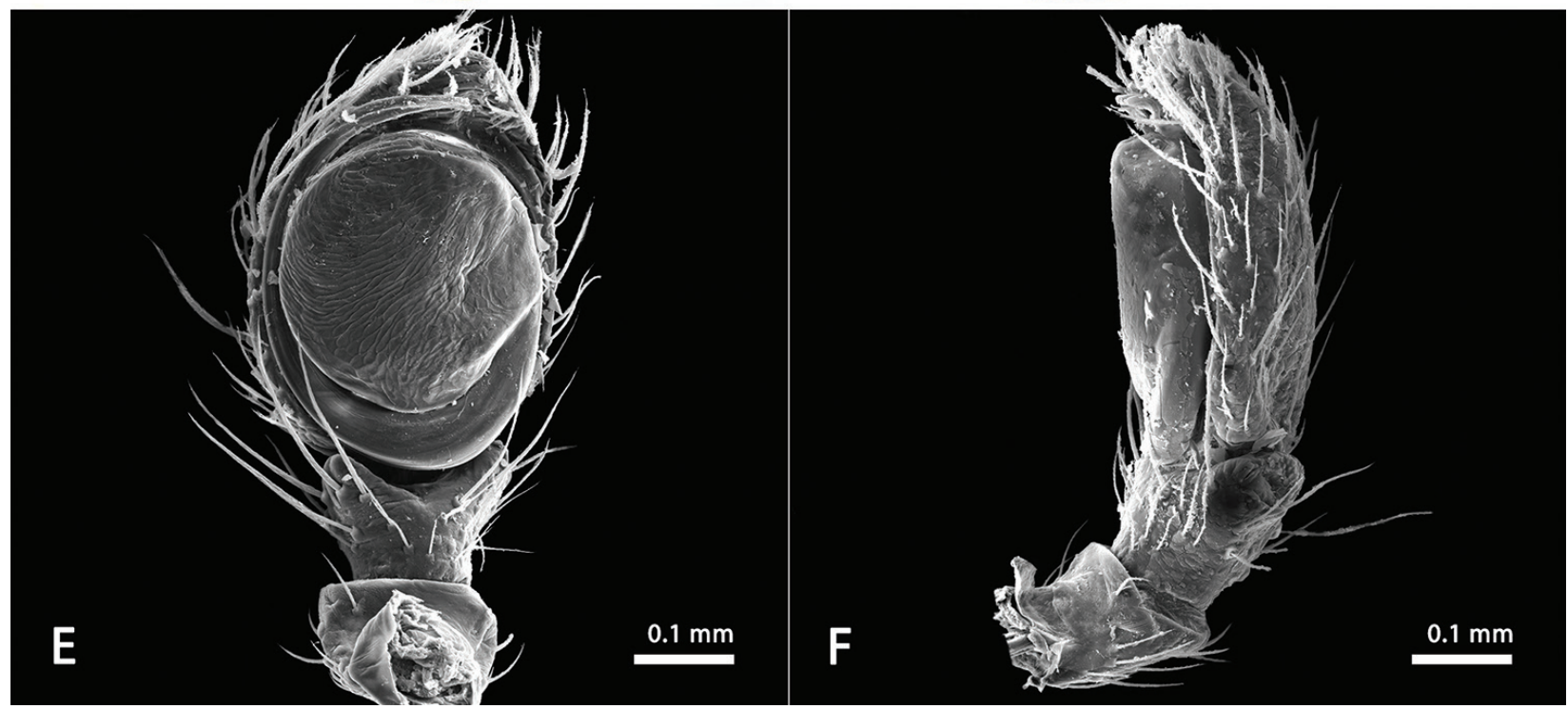

Figure 9. Male of Sidymella kolpogaster a habitus, dorsal b prosoma, anterior c, e left palp, ventral view d, f left palp, retrolateral view. 
Measurements: eyes diameters and eyes interdistances: AME 0.03, ALE 0.05, PME 0.03, PLE 0.05, AME-AME 0.11, AME-ALE 0.05, PME-PME 0.09, PME-PLE 0.09 , MOQ length 0.21 , MOQ width 0.11 ; leg formula: 1-2-4-3: leg I - femur 2.04/ patella 0.68 / tibiae 1.68 / metatarsus $1.56 /$ tarsus $0.60 /$ total 6.56 ; II $-1.64 / 0.36 /$ $1.44 / 1.20 / 0.52 / 5.16$; III $-0.72 / 0.36 / 0.52 / 0.52 / 0.32 /$ 2.44; IV $-0.92 / 0.32 / 0.76 / 0.48 / 0.36 / 2.84$. Total body length 3.12 ; prosoma 1.40 length, 1.32 wide; opisthosoma length 1.72; clypeus 0.07 height; sternum 0.64 length, 0.68 width; endites 0.27 length, 0.32 width; labium 0.13 length, 0.25 width.

Distribution. BRAZIL: Paraná, Rio Grande do Sul, Santa Catarina (Fig. 17).

\section{Sidymella longispina (Mello-Leitão, 1943)}

Figures 2A, 3A, 10, 11

Sidyma longispina Mello-Leitão, 1943: 208, fig. 35 (क); Lise 1973: 10, figs 19--23 ().

Type material. Holotype 9 , BRAZIL: Rio Grande do Sul, B. Rambo leg. (MNRJ 41911, examined; lost in the fire of the Museu Nacional do Rio de Janeiro). Neotype female, BRAZIL: Rio Grande do Sul, São Francisco de Paula, 29²4'52"S, 50¹5'24"W, A.A. Lise et al., 24-26. iv.2006 (MCTP 35899, designated here).

Other material examined. BRAZIL: $1 \hat{\jmath}$, Minas Gerais, Vale Verde (Parque Nacional do Caparaó), 20²5'05"S, 41 ${ }^{\circ} 50^{\prime} 48^{\prime \prime} \mathrm{W}, 24-30 . x i .2014$, B.T. Faleiro leg. (UFMG 18264). 10̄, 1j, Paraná, Foz do Iguaçu (Refúgio Biológico de Bela Vista), 25²6'56"S, 54³3'23"W, 09-11. xi.1991, A.B. Bonaldo leg. (MCN 21809). 19, Santa Catarina, Rancho Queimado, 2704'22"S, 4901'19"W, 08-12.x.1994, A.B. Bonaldo \& L.A. Moura leg. (MCTP 5952). 3j, Rio Grande do Sul, Tenente Portela, $27^{\circ} 22^{\prime} 15^{\prime \prime} \mathrm{S}$,

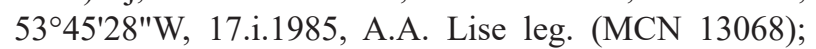
2j, Iraí, 27¹1'38"S, 53¹5'03"W, 18.xi.1975, A.A. Lise leg. (MCN 3142); 10j, same locality, 21.xi.1975, same collector (MCN 8082); 1j, Derrubadas (Parque Estadual do Turvo), 27²1'57"S, 5351'04"W, 04-06.ii.1980, A.A. Lise leg. (MCN 8984); 39, same locality, 0407.v.2004, R. Ott leg. (MCN 38864); 1ㅇ, same locality, 19-22.x.2004. R. Ott leg. (MCN 38878); 1f, Caxias do Sul (Vila Oliva), 29¹2'56"S, 5053'22"W, 14.ix.1976, H. Bischoff leg. (MCN 4485); 1ð̄, 10.iv.1992, L.A. Moura leg. (MCN 22124); 1j, Canela, 29²1'57"S, 5048'57"W, 24.viii.1975, A.A. Lise leg. (MCN 3026); 2 , , São Francisco de Paula, 29²7'00"S, 50³4'59"W, 14.v.1993, A. Braul leg. (MCTP 3213); 3j, same locality, 05.i.1985, A.A. Lise leg. (MCN 13107); 1 q , Maquiné, 2940'47"S, 50¹1'20"W, 22.vi.2008, E.N.L. Rodrigues leg. (MCN

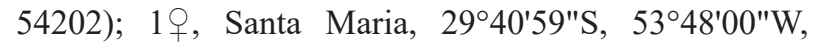
18.i.1999, C.B. Kotzian \& L. Indrusiak leg. (MCTP 40101); 1ð, 1ㅇ, same locality, 30.ix.1992, biology students leg. (MCTP 41330); 19, Gravataí (Mato Alto),
2957'20"S, 5057'46"W, 13.iii.1985, A.D. Brescovit leg. (MCN 13094); 2 , , Cachoeira do Sul, 300'0"S, $53^{\circ} 0^{\prime} 0^{\prime \prime} \mathrm{W}, \mathrm{B}$. Rambo leg. (MNRJ 41911); 1 \%, Pantano Grande, 30¹1'27"S, 52²2'26"W, 05.iv.2008, G. Deprá leg. (MCTP 40084); 1은 Guaíba, 306'50"S, 5119'30"W, 28.iv.1995, A. Braul leg. (MCTP 7542); 19 , Cristal (Rio Camaquã), $31^{\circ} 00^{\prime} 12^{\prime \prime S}, 52^{\circ} 04^{\prime} 02^{\prime \prime} \mathrm{W}$ 19.xii.2007, E.N.L. Rodrigues leg. (MCN 48618); 1ठ̄, same locality, 14.ii.2008, same collector (MCN 48832); 1 ㅇ, same locality, 21.iv.2008, same collector (MCN 49031); 1j, São Borja, 28¹0'16.10"S, 55²6'49.78"W, 06.xii.1975, A.A. Lise leg. (MCN 8088). ARGENTINA: $1 \bigcirc$, Misiones, Puerto Iguazu (Parque Nacional Iguazú), 2541'01"S, 54²7'14"W, 22-30.viii.1986, M. Ramírez leg. (MACN-Ar 19069); 2j, same locality, 19-20.i.2005, C. Grismado et al. leg. (MACN-Ar 27637); 1 ㅇ, same locality, vi.1985, M. Ramírez leg. (MACN-Ar 19067); 1j, Iguazu, 2550'53.71"S, 54²0'48.17"W, x.1954, Schiapelli de Carlo leg. (MACN-Ar 19070); 1j, Monte-

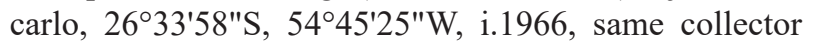
(MACN-Ar 19072); 3ð̄, 2j, Santa Maria, 2753'39"S, 5521'20"W, 1952, C. Viana leg. (MCTP 3537).

Diagnosis. Females of $S$. longispina are similar to those of $S$. furcillata by their long and vertically oriented opisthosomal projections (Figs 2A, 10A), and the presence of stout macrosetae above the ALE (Fig. 10B); however, their opisthosomal projections have pointy conical apexes instead of being rounded with a small apical protuberance as in $S$. furcillata. The females of S. longispina also have shorter copulatory ducts (Fig. 10D, F). Males of $S$. longispina resemble those of $S$. furcillata but their palpi bear a truncated RTA with a conical and well-developed basal branch (larger than the RTA itself) (Fig. 11D, F); the male palp has a long, acute tegular process and a dorso-basal projection (Fig. 11D, F). Both males and females have just one mesial macroseta on femora I (Fig 11A).

Description. Female: Prosoma yellowish-orange, darker on the cephalic area and presenting a pair of needle-shaped macrosetae on conical projections above the ALE (Fig 10B). Legs yellowish-orange; femora I with a single prolateral macrosetae; both the anterior tibiae and metatarsi (I and II) ventrally armed with five pairs of ventral macrosetae. Opisthosoma light-yellow with posterior projections long, pointed and vertically oriented (Figs 2A, 10A).

Measurements: eyes diameters and eyes interdistances: AME 0.05, ALE 0.09, PME 0.05, PLE 0.05, AME-AME 0.15, AME-ALE 0.11, PME-PME 0.15, PME-PLE 0.17, MOQ length 0.35 , MOQ width 0.15 ; leg formula: 1-2-43: leg I - femur 4.45/ patella 1.60/ tibiae 4.20/ metatarsus $3.05 /$ tarsus $0.85 /$ total 14.15 ; II $-3.35 / 1.30 / 2.95 / 1.95 /$ $0.75 / 10.30$; III - $1.25 / 0.65 / 1.05 / 0.55 / 0.55 / 4.05$; IV $1.60 / 0.70 / 0.95 / 0.55 / 0.45 / 4.25$. Total body length 7.93 ; prosoma 2.56 length, 2.12 wide; opisthosoma length 5.37 (considering the projections); clypeus 0.23 height; sternum 1.11 length, 1.00 width; endites 0.60 length, 0.27 width; labium 0.31 length, 0.37 width. 

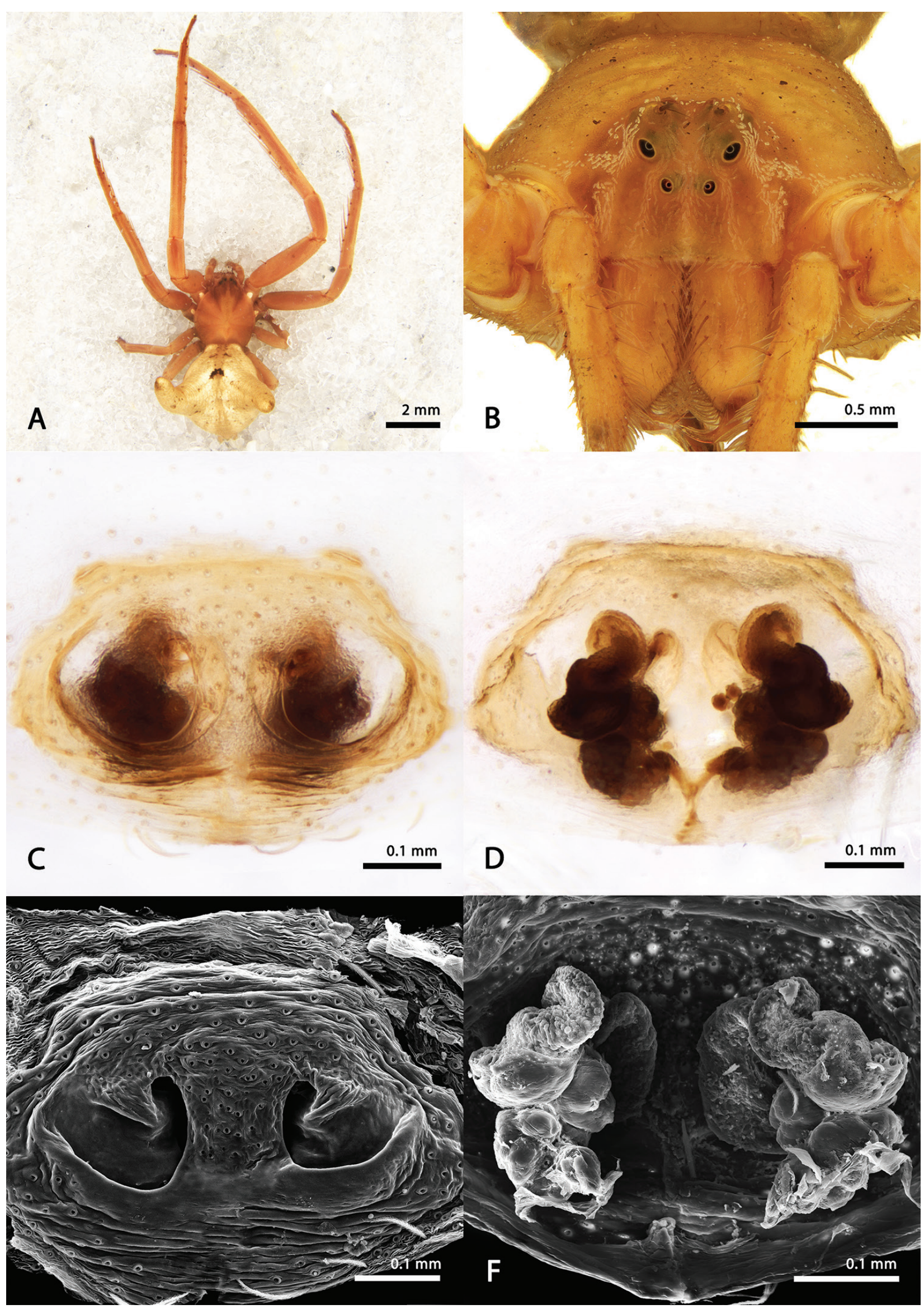

Figure 10. Female of Sidymella longispina a habitus, dorsal b prosoma, anterior c, e epigynum, ventral d, f epigynum, dorsal. 


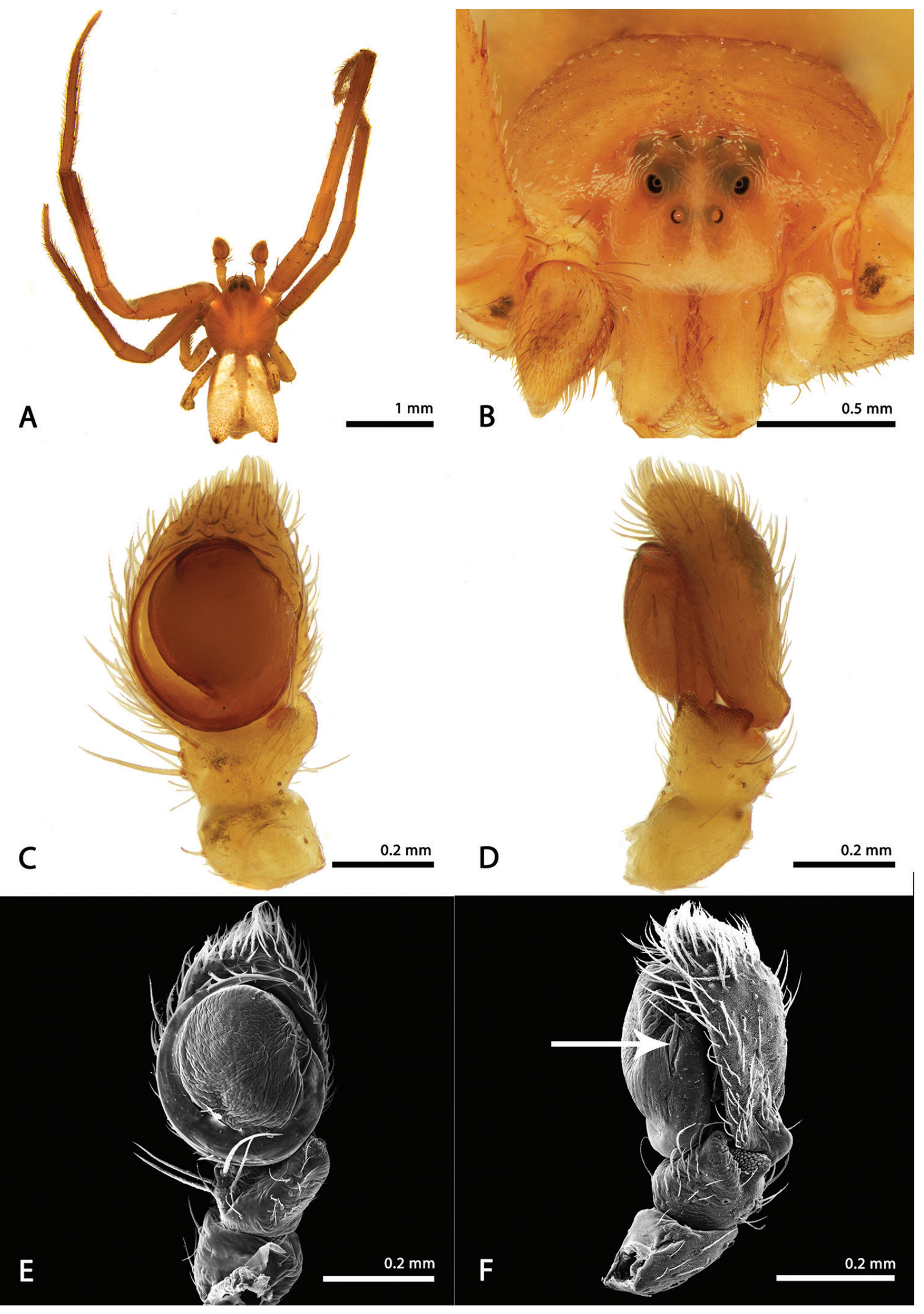

Figure 11. Male of Sidymella longispina a habitus, dorsal b prosoma, anterior c, e left palp, ventral view $\mathbf{d}$, $\mathbf{f}$ left palp, retrolateral view (white arrow indicates the tegular process). 
Male: Body colouration pattern and other characteristics as in female (Fig. 11A, B).

Measurements: eyes diameters and eyes interdistances: AME 0.03, ALE 0.05, PME 0.03, PLE 0.03, AME-AME 0.09, AME-ALE 0.05, PME-PME 0.11, PME-PLE 0.11, MOQ length 0.21 , MOQ width 0.13 ; leg formula: $1-2-4$ 3: leg I - femur 2.44/ patella 1.12/ tibiae 3.12/ metatarsus 2.28/ tarsus $0.92 /$ total 9.88; II $-2.08 / 0.76 / 1.64 / 1.48 /$ $0.72 / 6.68$; III - 0.60/ 0.48/ 0.60/ 0.40/ 0.40/ 2.48; IV $1.04 / 0.44 / 0.60 / 0.40 / 0.40 / 2.88$. Total body length 3.04 ; prosoma 1.40 length, 1.72 wide; opisthosoma length 1.64 (considering the projections); clypeus 0.17 height; sternum 0.76 length, 0.66 width; endites 0.33 length, 0.17 width; labium 0.17 length, 0.25 width.

Distribution. BRAZIL: Minas Gerais, Paraná, Santa Catarina, and Rio Grande do Sul; ARGENTINA: Misiones (Fig. 17).

\section{Sidymella lucida (Keyserling, 1880)}

Figures 2F, 3E, 12, 13

Stephanopis lucida Keyserling, 1880: 190, pl. 4, fig. 105 (†).

Sidyma lucida: Simon 1895: 1056. Berland 1913: 95, pl. 9, figs 51-56 ( $\overbrace{}^{\lambda}+$ )

Sidyma cancellata: Mello-Leitão 1934: 207, fig. 34 (ठ).

Sidymella lucida: Strand 1942: 399. Machado et al. 2017: 454, figs S11A, F, S14B (ठ).

Sidyma lucida Lise, 1973: 6, figs 10-18 (ぷ゚).

Type material. Holotype $\hat{\jmath}$, BRAZIL: Rio Grande do Sul, B. Rambo (MNRJ 41748, examined).

Other material examined. ECUADOR: $1 \hat{\jmath}$, Tunguhua, Baños, 01²3'51"S, 78²5'27"W, 10.iv.1939 (MCZ 133401); 1ô, 1, 2j, same locality, iv.1939 (MCZ 133402); COLOMBIA: 19 , Valle del Cauca, Cali, 320'50"N, 76³4'34"W, x.1972 (MCZ), BRAZIL: 1의, Minas Gerais, Tiradentes (Serra de São José), $21^{\circ} 06^{\prime} 36^{\prime \prime S}$, 4410'23"W, 28.x.1989, Becker, Johann \& Roppa leg. (MNRJ 2694); 19 , Rio de Janeiro, Santa Maria Madalena, 21 ${ }^{\circ} 57^{\prime} 18^{\prime \prime S}, 42^{\circ} 0^{\prime} 28^{\prime \prime} \mathrm{W}, 15 . v .2002$ (MCN 34671); 1q, São Paulo, Vale do Ribeira, 24³7'28.68"S, 47²3'33.99"W, vi.2002, E.H. Wienskoski leg. (MNRJ 11513); 1 + , 1j, Paraná, São José dos Pinhais, 25³2'06"S, 49¹2'21"W, 09.xii.2015, A.C. Domahovski leg. (MCTP 39092); 1ภ, 2ㅇ, Santa Catarina, Rancho Queimado, $27^{\circ} 40^{\prime} 22^{\prime \prime S}, 49^{\circ} 01^{\prime} 19^{\prime \prime W}, 13-15 . i .1995$, L.A. Moura leg. (MCN 26465); 1 ㅇ, Alta Feliz, 29²3'31"S, 5118'43"W, vii.1988, A.A. Lise leg. (MCTP 526); 1 \%, Rio Grande do Sul, Derrubadas (Parque Estadual do Turvo), 27 $13^{\prime} 57^{\prime \prime S}$, 5351'04"W, 27-31.x.2003, R. Ott leg. (MCN 37826); 19 , same locality, 19-22.x.2004, same collector (MCN 38879); 1 q, 1j, São Valentim, 27³3'28"S, 52³1'26"W, 16.x.1976 (MCN 4704); 1ㅇ, Santa Rosa, 2752'15"S, 54²8'34"W, 02.i.1984, A.D. Brescovit leg. (MCN 11989); 2j, Esmeralda, 2803'14"S, 51 ${ }^{\circ} 11^{\prime} 24^{\prime \prime} \mathrm{W}$, 24.v.1975, A.D. Brescovit leg. (MCN 2881); 1 , , Esmer- alda (Estação Ecológica de Esmeralda), 2803'8.35"S, 511'36.92"W, 12.xii.1978, C.J. Becker leg. (MCN

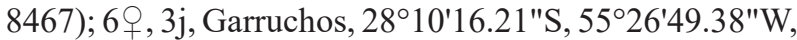
08.xii.1975, A.A. Lise leg. (MCN 8083); 1 ㅇ, same locality, 08.xi.1979, H. Bischoff leg. (MCN 8965); 4j, Vacaria, $28^{\circ} 30^{\prime} 43^{\prime \prime S}, 50^{\circ} 56^{\prime} 02^{\prime \prime} \mathrm{W}, 23 . v .1981$ (MCN 9758); 1ठ, Augusto Pestana, 28³1'01"S, 5359'31"W, 06.ix.2009, L.V. Silva-Leomar \& B. Medeiros (MCTP 30588) 1 , same locality, 12.ix.2008, L.V. Silvia-Leomar et al. leg. (MCTP 27094); 1 , São Borja (Reserva Biológica de São Donato), 28³9'39"S, 5600'14"W, 23.i.2012, Machado, M. leg. (MCTP 34729); $2 \hat{\jmath}, 3$, , Salto do Jacuí (Horta da CEEE), 2905'21.03"S, 53¹2'41.24"W, 19.x.1998, A.B. Bonaldo leg. (MCN 30761); 19 , Caxias do Sul (Fazenda Souza), 2907'17"S, 5101'07"W, 11-12.xi.1995, lab staff leg. (MCTP 7322); 10̂, Muçum, 2909'54"S, 5152'04"W, 02.iii.1984 (MCN 12090); 1q, Caxias do Sul (Água Azul), 29¹1'51"S, 5059'27"W, 15.ix.1976, E.H. Buckup (MCN 4498); 1ठ, 4j, Caxias do Sul (Vila Oliva), 29¹2'56"S, 5053'22"W, 05.iv.1975, H. Bischoff leg. (MCN 2872); 1 , Estrela Velha (Barragem de Itaúba), 29॰15'19"S, 5313'36"W, 20.x.1998, L.A. Moura leg. (MCN 30763); 1 을 same locality, 28.x.1999, A. Silva (MCN 31959); 10̄, São Francisco de Paula, 29¹6'29"S, 5044'15"W, 19.xi.1990, E.H. Buckup leg. (MCN 28841); 1 을 same locality, 16.xii.1999, A.F. Franceschini leg. (MCN 32047); 1j, Canela, 29²1'57"S, 5048'57"W, 07.x.1967, A.A. Lise leg. (MCN 649); 3j, same locality, 05.ii.1970, same collector (MCN 651); 2 , , same locality, 20.i.1972, same collector (MCN 1025); 1 , , same locality, 31.xii.1973, same collector (MCN 2029); 1 으, same locality, 21.ix.1974, same collector (MCN 2249); 3 ㅇ, same locality, 26.xii.1974, same collector (MCN 2492); 1j, same locality, 08.xi.1975, same collector (MCN 5968); 1j, same locality, 05.i.1973, same collector (MCN 9056); 1j, same locality, 11.i.1966, same collector (MCN 9057); 1 + , São Francisco de Paula (FLONA), 29² $5^{\prime} 47^{\prime \prime S}$, 50²3'35"W, 19.xii.2010, R.A. Teixeira leg. (MCTP 33303); 1, same locality, 10.x.2012, same collector (MCTP 41327); 3j, São Francisco de Paula, 29²7'00"S, 50³4'59"W, 05.i.1985, A.A. Lise leg. (MCN 12723); 1j, Três Coroas, 29॰30'55"S, 5046'46"W, 15.xii.1976, A.A. Lise leg. (MCN 4924); 19, São Martinho da Serra, 29³2'16"S, 5351'18"W, 19.x.1993, L. Indrusiak \& R.A. Boelter leg. (MCTP 40116); 1ㅇ, Itaara, 29³6'36"S, 5345'54"W, 22.xi.2006, L. Indrusiak \& R.A. Boelter leg. (MCTP 21356); 2j, same locality, 23.vi.2007, A.A. Lise et al. leg. (MCTP 21357); 1j, same locality, 14.vii.2007, L. Indrusiak leg. (MCTP 21358); 1 + , Agudo, 29³8'31"S, 5315'10"W, 21.x.1988, L. Indrusiak leg. (MCN 18751); 1ㅇ, Campo Bom, 2940'44"S, 51³'10"W, 19.x.1987, L. Indrusiak leg. (MCTP 135); 1 , , Santa Maria, 2940'59"S,

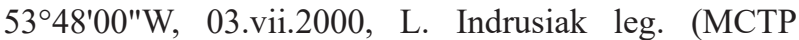
40092); 29, same locality, 29.vi.1998, same collector (MCTP 40094); 1ㅇ, same locality, 24.iii.1992, same collector (MCTP 40122); 1ð̄, same locality, 28.vii.1995, C.B. Kotzian \& L. Indrusiak leg. (MCTP 40100); 1 웅, same locality, 14.x.2004, C.B. Kotzian leg. (MCTP 
40091); 19, same locality, 03.iv.2013, L. Indrusiak leg. (MCTP 39419); 1ðิ, 19, same locality, 24.v.2007, A. Aued \& E. Felzmamm leg. (MCTP 40103); 1, 30. vii.2000, L. Indrusiak leg. (MCTP 40090); 1 , , same locality, 15.vii.1998, C.B. Kotzian \& L. Indrusiak leg. (MCTP 40102); 1옹, same locality, 22.v.1996, same collector (MCTP 40097); 19, same locality, 20.x.1995, same collector (MCTP 40098); 3ô, 1ㅇ, same locality, 19.vi.1998, C. Kotzian \& M. Monteiro leg. (MCTP 41324); 1 ㅇ, same locality, 17.xii.1992, L. Indrusiak \& M. Monteiro leg. (MCTP 41323); 1, same locality, 21. xii.1993, L. Indrusiak leg. (MCTP 41322); 1ㅇ, same locality, 17.iii.1998, C. Kotzian \& L. Indrusiak leg. (MCTP

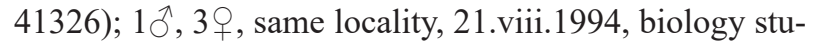
dents leg. (MCTP 41325); 1ð̄, 1q, same locality, 26. viii.1992, E. Lang \& L. Oleques leg. (MCTP 40096); $3 \hat{\jmath}$, 1 , , same locality, 30.ix.1998, L. Indrusiak \& M. Monteiro leg. (MCTP 40095); 1ð̄, same locality, 15.x.1998, C. Kotzian \& L. Indrusiak leg. (MCTP 40104); 1j, same locality, 10.x.1979, D. Link leg. (MCN 9548); 3 q, same locality, 02.xi.1985, A.D. Brescovit leg. (MCN 14565); 20̄, same locality, 31.viii.1994, R.G. Buss leg. (MCTP 10254); 5j, Montenegro, 2941'20"S, 51²7'39"W, 01. ix.1977 (MCN 6362); 1j, same locality, 06.x.1977, T. Arigony leg. (MCN 6808); 1j, same locality, 29.ix.1977, A.A. Lise leg. (MCN 8180); 10, Santa Cruz do Sul, 294'04"S, 52²5'33"W, 14.viii.1994, A.A. Lise leg. (MCN 33985); 1ð̄, Novos Cabrais, 2946'59"S, 52॰58'18"W, 01.xi.2008, R.G. Buss leg. (MCTP 27971); $1 \hat{O}^{\top}$, same locality, 11.ix.2008, same collector (MCTP 28090); 1j, Alegrete, 2947'26"S, 5547'43"W, 28. viii.1984, M.A.L. Marques leg. (MCN 9717); 1ठ, Morungava, 2951'11"S, 5054'31"W, 02.ii.1992, A.D. Brescovit leg. (MCN 23177); 1ð, 3ㅇ, Cachoeira do Sul (Porteira 7), 3001'18.65"S, 52॰55'3.70"W, 31.x.1992, R.G. Buss leg. (MCTP3485); 19, 1j, same locality, 26. vii.1992, same collector (MCTP 3488); 1우, Porto Alegre, $30^{\circ} 01^{\prime} 58^{\prime \prime S}$, 51²13'48"W, 01.xi.2009, I. Martins leg. (MCTP 26258); 1ð̄, 2ᄋ, 1j, Cachoeira do Sul, 3001'59"S, 52 54'00"W, 10.x.1992, R.G. Buss leg. (MCTP 3491); $1 \hat{\jmath}, 1$, , same locality, 26.ix.1992, same collector (MCTP

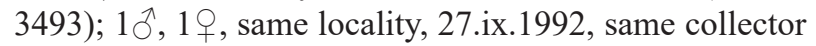
(MCTP 3487); 1j, Porto Alegre (Morro Santana), 3002'34"S, 5108'39"W, 01.ix.1984, A.A. Lise leg. (MCN 12546); 1j, Viamão (Estação Fitotécnica de Viamão), 3002'51"S, 5100'53"W, 12.viii.1994, A.A. Lise leg. (MCTP 5245); 1j, same locality, 30.iii.1976 same collector (MCN 5860); 1ठ, Viamão, 3004'51"S, $51^{\circ} 01^{\prime 2} 2$ "W, 22.xi.1995, A.A. Lise leg. (MCTP 12246); 1ðં, Guaíba, 3006'50"S, 51¹9'30"W, 04.x.1995, (MCTP 12334); 1ㅇ, São Sepé, 3009'50"S, 53³4'18"W, iv.1999, E.C. Costa leg. (MCTP 40099); 1ð̃, same locality, 03.x.2002, C.B. Kotzian leg. (MCTP 41321); 1웅, Cachoeira do Sul (Fazenda das Pedras), 30¹2'50"S, 52॰50'21"W, 27.x.1992, R.G. Buss leg. (MCTP 3490); 1ㅇ, same locality, 09.ix.1992, same collector (MCTP 3486); 1ð,, same locality, 30.xi.1993, same collector (MCTP 4251); 1을 Cachoeira do Sul (Capanezinho),
30¹8'35"S, 5258'50"W, 29.viii.1992, R.G. Buss leg. (MCTP 3492); 1ð̄, 2ㅇ, same locality, 17.x.1992, same collector (MCTP 3489); 2 , Viamão (Morro Fortaleza), 30²0'45"S, 51 01'35"W, 22.xi.1992, A. Braul leg. (MCTP 2656); 2j, Quaraí, 30²3'03"S, 56²6'56"W, 24 28.v.1991, A. Braul leg. (MCTP 461); 4j, Quaraí (Estância São Roberto), 30²5'14"S, 5551'53"W, 07.ii.1978, J.W. Thomé leg. (MCN 7779). ARGENTINA: $7 \hat{\jmath}, 3 q$, Jujuy, Parque Nacional Calilegua, 2338'20"S, 643'ㄱ'"W, 23-24.ix.1995 (MACN-Ar 19229); 10̄, Misiones, El Piñalito, 2558'59"S, 5353'59"W, xi.1954, Schiapelli di Carlo leg. (MACN-Ar 19095); 19, Tucuman, Racó, 263' $15^{\prime \prime S}, 65^{\circ} 22^{\prime} 43^{\prime \prime W}$ (MACN-Ar 19102); 1j, Arroyo Yabebiry (Ruta Nacional 12),

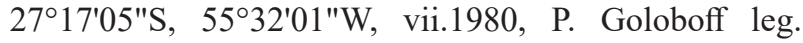
(MACN-Ar 19100); 19, Santa Maria, 2753'39"S, 55²1'20"W, vii.1954, M.J. Viana leg. (MACN-Ar 19097); 1 , , same locality, x.1953, M.J. Viana \& Schiapeli de Carlo leg. (MACN-Ar 3804); $3 \hat{\jmath}$, same locality, xii.1952, J. Viana leg. (MACN-Ar 3535); 1j, same locality, xi.1952, J. Viana leg. (MACN-Ar 19071); 1j, same locality, 1954, M. Belgrano \& Schiapelli de Carlo leg. (MACN-Ar 19096); 1 9, same locality, xii.1947, J. Viana leg. (MACN-Ar 2455); 2 , same locality, xi.1952, J. Viana leg. (MACN-Ar 3534); 1今̄, Entre Ríos, Rosario del Tala, 32॰18'00"S, 5908'00"W, 20.xi.1988, M. Ramírez leg. (MACN-Ar 19091). URUGUAY: 19 , Lavalleja,

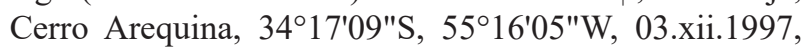
A.A. Lise leg. (MCTP 12677).

Diagnosis. Females of $S$. lucida resemble those of $S$. excavata sp. nov. and $S$. marmorata sp. nov. by their short and rounded opisthosomal projections (Figs 2F, 12A). They can be distinguished by shorter copulatory ducts and spermathecae with just a median twisted constriction instead of many chambers (Fig. 12D, F). Males are similar to those of $S$. excavata sp. nov. by the colour pattern of the opisthosoma and the shape of its posterior projections. However, males of $S$. lucida have a roundish RTA with a discrete basal branch (Fig. 13D, F), narrower pars pendula, and a shorter embolus emerging from tegulum at five o'clock (Fig. 13C, E).

Description. Female: Prosoma yellowish, with cephalic ridges delimited by lines of small papules; needle-shaped setae concentrated on the median area of prosoma, being the largest ones disposed right back of the PLE (Fig. 12B). Legs yellowish, with femora I bearing three equalsized needle-shaped macrosetae on their mesial surface (Fig. 12A); both the anterior tibiae and metatarsi (I and II) ventrally armed with five pairs of ventral macrosetae; tibiae I also bear a pair of smaller macrosetae along their mesial surface (Fig. 12A). Opisthosoma light-yellow with a median black stain on its anterior portion; posterior projections stout, obtuse and vertically oriented (Fig. 12A). Epigynal plate wide, depressed on the median field; posterior folds thick and converging in the middle to form a septum (Fig. 12C, E). 

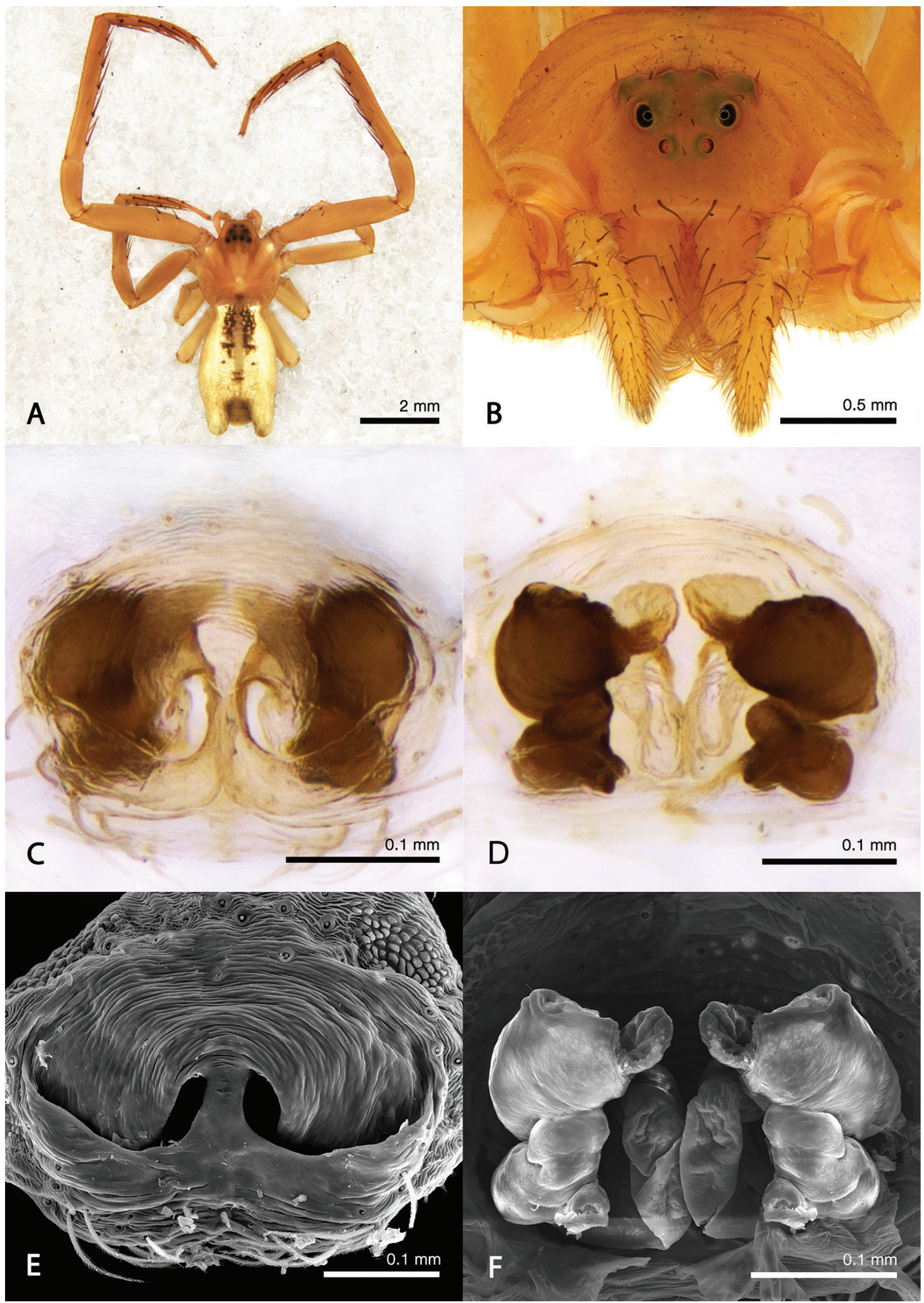

Figure 12. Female of Sidymella lucida a habitus, dorsal b prosoma, anterior c, e epigynum, ventral d, f epigynum, dorsal. 

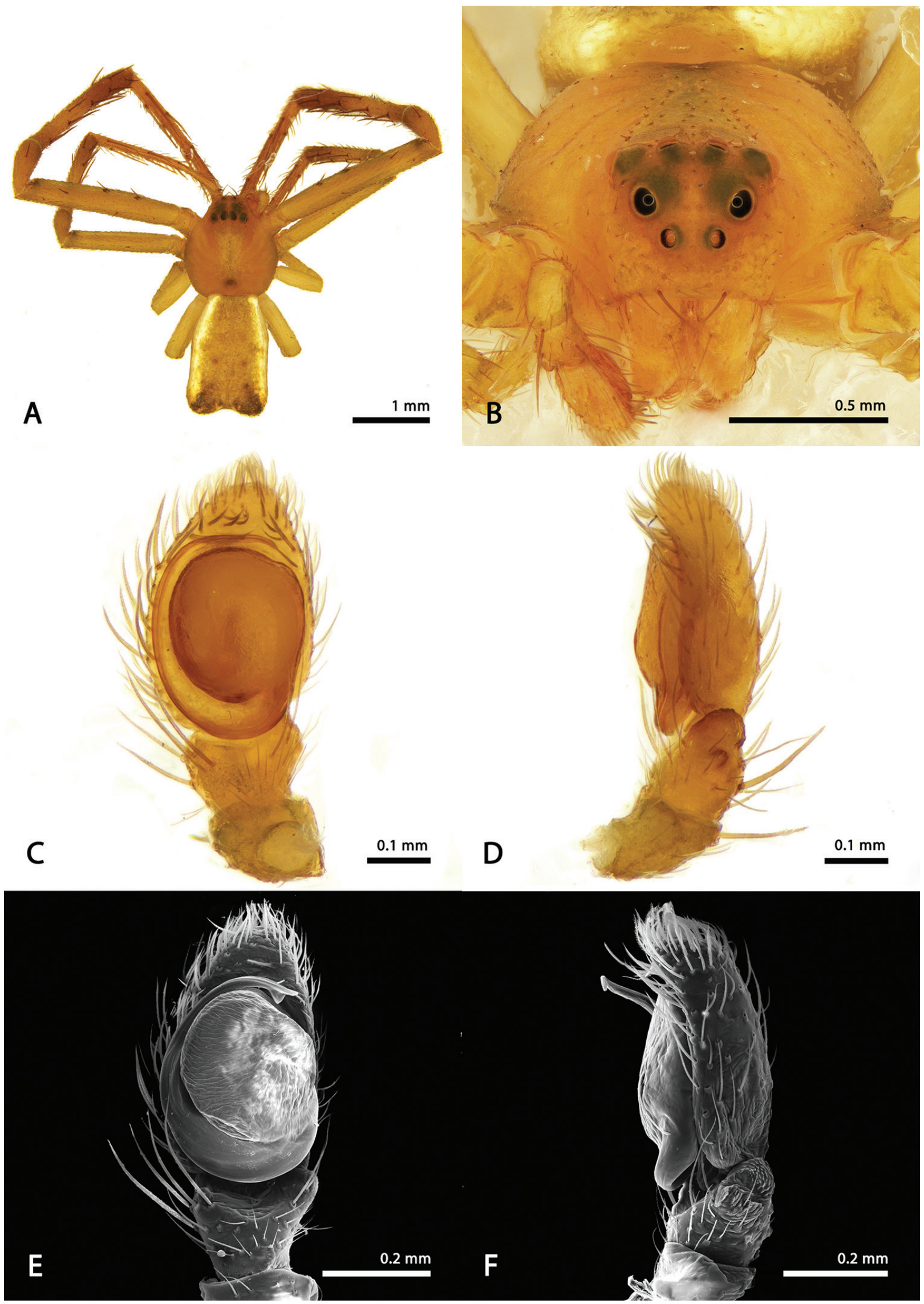

Figure 13. Male of Sidymella lucida a habitus, dorsal b prosoma, anterior c, e left palp, ventral view d, f left palp, retrolateral view (black arrow indicates the basal branch of the RTA). 
Measurements: eyes diameters and eyes interdistances: AME 0.05, ALE 0.07, PME 0.05, PLE 0.05, AME-AME 0.11, AME-ALE 0.05, PME-PME 0.09, PME-PLE 0.17, MOQ length 0.29 , MOQ width 0.23 ; leg formula: $1-2-4$ 3: leg I - femur 3.10/ patella 1.28/ tibiae 3.16/ metatarsus 2.32/ tarsus 0.84 / total 10.70 ; II $-2.00 / 0.96 / 1.64 / 1.48 /$ $0.56 / 6.64$; III $-0.80 / 0.52 / 0.68 / 0.44 / 0.40 / 2.84$; IV $1.04 / 0.60 / 0.68 / 0.52 / 0.36 / 3.20$. Total body length 4.68 ; prosoma 1.96 length, 1.76 wide; opisthosoma length 2.72 (considering the projections); clypeus 0.27 height; sternum 1.08 length, 0.84 width; endites 0.44 length, 0.28 width; labium 0.28 length, 0.32 width.

Male: Prosoma and legs as in female; opisthosoma predominantly yellow with lateral whitish lines, posterior projections dark (Fig. 13A, B). Palp flattened dorsoventrally and elongated; cymbium distally narrowed; tegulum rounded with scaled surface (Fig. 13C-F).

Measurements: eyes diameters and eyes interdistances: AME 0.05, ALE 0.07, PME 0.05, PLE 0.05, AME-AME 0.11, AME-ALE 0.05, PME-PME 0.09, PME-PLE 0.17, MOQ length 0.29, MOQ width 0.23; leg formula: 1-2-4-3: leg I - femur 2.56/ patella 0.88 / tibiae $2.48 /$ metatarsus $2.12 /$ tarsus $0.72 /$ total 8.76 ; II $1.68 / 0.60 / 1.36 / 1.28 / 0.56 / 5.48$; III - 0.64/ 0.32/ 0.56/ $0.28 / 0.36 / 2.16$; IV $-0.84 / 0.44 / 0.48 / 0.24 / 0.36 / 2.36$. Total body length 3.00 ; prosoma 1.40 length, 1.20 wide; opisthosoma length 1.60 (considering the projections); clypeus 0.15 height; sternum 0.58 length, 0.76 width; endites 0.27 length, 0.13 width; labium 0.17 length, 0.15 width.

Distribution. ARGENTINA: Entre Rios, Jujuy, Missiones, and Tucumán. BRAZIL: Paraná, Rio de Janeiro, Rio Grande do Sul, and Santa Catarina. COLOMBIA: Cáli and Nova Granada. ECUADOR: Loja and Tungurahua. URUGUAY: Lavalleja (Fig. 16).

\section{Sidymella marmorata Machado \& Guzati, sp. nov.} http://zoobank.org/0EB278EC-9D36-4D68-8D52-9CE838B66501 Figures 2E, 14

Type material. Holotype $q$, ECUADOR: Quito (Road to Santo Domingo), 0¹9'3.83"S, ${ }^{\circ} 59^{\prime} 19.95^{\prime \prime W}$, L. Pena leg. (MCZ 133399). Paratypes. ECUADOR: 1 ㅇ, Quito (Road to Santo Domingo), 0¹9'3.83"S, ${ }^{\circ} 59^{\prime} 19.95^{\prime \prime W}$, 24.ii.1965, L. Pena leg. (MCTP 42654); COLOMBIA: 1 q, Cundinamarca (Chía, Alto de Yerbabuena), $4^{\circ} 51^{\prime} 53.13^{\prime \prime N}, 74^{\circ} 3^{\prime} 3.30^{\prime \prime} \mathrm{W}, 09 . x .2005$, K. Lara \& X. Marquínez leg. (ICN 7578)

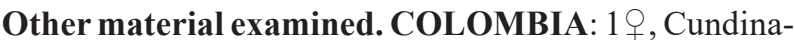
marca, Zipacón, 044 45'44"N, 74²2'46"W, 01.i.2011, D. Luna \& C. Romero leg. (ICN-Ar 6379); 1 + , 1j, Boyacá, 0541'14"N, 73²6'9"W, 01.viii.2003 (MPUJ_ENT);

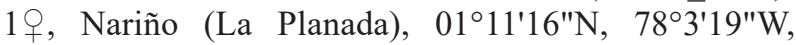
29.ii.1991, C. Valderrama leg. (LPN 226); 1 오, Valle del Cauca (Chicoral), 03³9'24"N, 76²41'18"W, iii.2005,
J. Cabra leg. (MUSENUV 28231); 19 , Quindío (Buenavista), $04^{\circ} 21^{\prime} 59^{\prime \prime} \mathrm{N}, 7^{\circ} 44^{\prime} 37^{\prime \prime} \mathrm{W}, 13.1 i .2010$ (CIUQ 9663); 19, Cundinamarca (Universidade Javeriana), 04³7'44"N, 7403'51"W, 03.xi.1995 (IAvH 1157); 1 웅, Quindío (Bengala), 0440'0"N, 75³9'0"W, 05.v.2003, A. Ardila leg. (MAUQ 1691); 39 , 2j, Cundinamarca (Alto de Yerbabuena), 0451'55"N, 7401'27"W, ix.2005, K. Lara \& X. Marquínez leg. (ex. ICN-Ar 7578); 1 \% , Cundinamarca (Suesca), $05^{\circ} 06^{\prime} 12^{\prime \prime N}$, 7347'56"W, 06.x.2013 (ICN-Ar 7637); 19, Caldas (Samaná), 05³6'0"N, 75²'0"W, 23.xi.1995, V. Rueda \& H. Piñeros leg. (IAvH 180518); 1 , , Boyacá, (Villa de Ley-

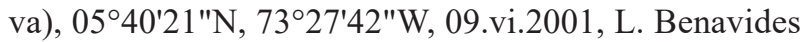
\& J. Pinzón leg. (ICN-Ar 1249).

Etymology. The epithet means marbled or overlaid with marble and refers to the reticulated colour pattern of the opisthosoma.

Diagnosis. Females of $S$. marmorata sp. nov. resemble those of $S$. excavata sp. nov. by the large body size and the general shape of opisthosoma with an anterior concavity and short posterior projections (Figs 2E, 14A). However, females of $S$. marmorata sp. nov. bear numerous spiniform macrosetae on the mesial surface of femora I and II and five, instead of four, pairs of ventral macrosetae on tibiae I and II (Fig. 14A).

Description. Female: Prosoma and legs I and II entirely orange while the posterior legs (III and IV) are yellowish. Opisthosoma predominantly yellow but with brownish irregular stains distributed randomly, giving a "marbled" aspect to the spider's dorsum (Fig. 14A); opisthosoma projections short and stout and anal region projected backwards (Figs 2E, 14A). Epigynal plate elevated, with a wide septum and lateral folds concentrically developed towards to the copulatory openings (Fig. 14C, E) copulatory ducts long, hyaline and coiled, leading to a pair of walnut-shaped spermathecae subdivided in chambers and with a tubular glandular-head (Fig. 14D, F).

Measurements: eyes diameters and eyes interdistances: AME 0.07, ALE 0.11, PME 0.07, PLE 0.07, AME-AME 0.17, AME-ALE 0.13, PME-PME 0.19, PME-PLE 0.19 , MOQ length 0.37 , MOQ width 0.17 ; leg formula: 1-2-4-3: leg I - femur 3.20/ patella 1.20/ tibiae 2.85/ metatarsus 1.80 / tarsus $0.88 /$ total 9.93 ; II $-2.40 / 1.03$ / 2.12/ 1.60/ 0.77/ 7.92; III - 1.40/ 0.76/ 0.92/ 0.84/ 0.44/ 4.36; IV - 1.72/ 0.76/ 1.08/ 0.92/ 0.52/ 5.00. Total body length 5.44; prosoma 2.36 length, 2.32 wide; opisthosoma length 3.08; clypeus 0.33 height; sternum 1.03 length, 1.07 width; endites 0.58 length, 0.23 width; labium 0.33 length, 0.41 width.

Male: Unknown.

Distribution. ECUADOR: Quito. COLOMBIA: Cundinamarca, Boyacá, Nariño, Valle del Cauca, and Quindío (Fig. 15). 

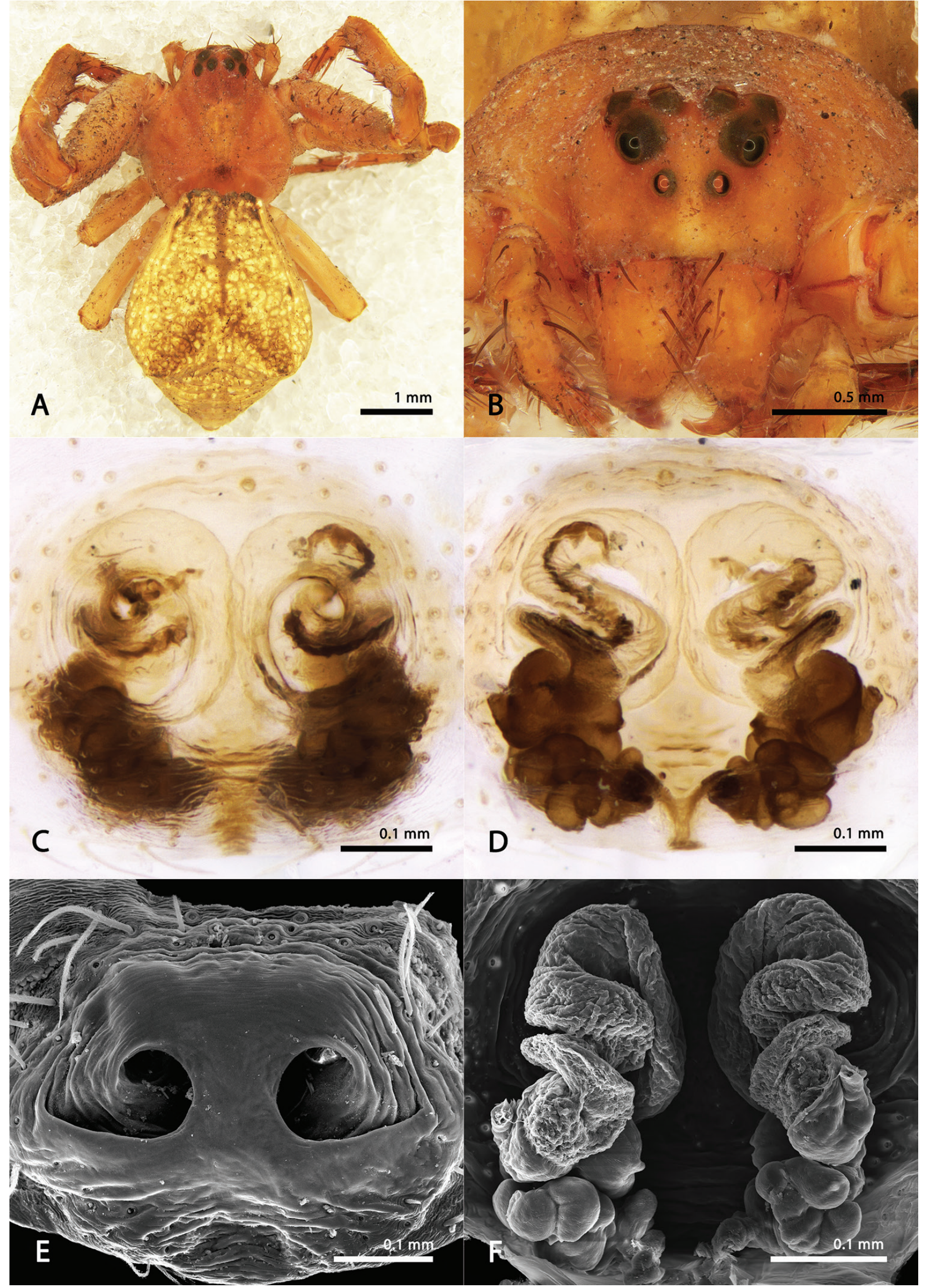

Figure 14. Female of Sidymella marmorata sp. nov. a habitus, dorsal b prosoma, anterior c, e epigynum, ventral d, $\mathbf{f}$ epigynum, dorsal. 


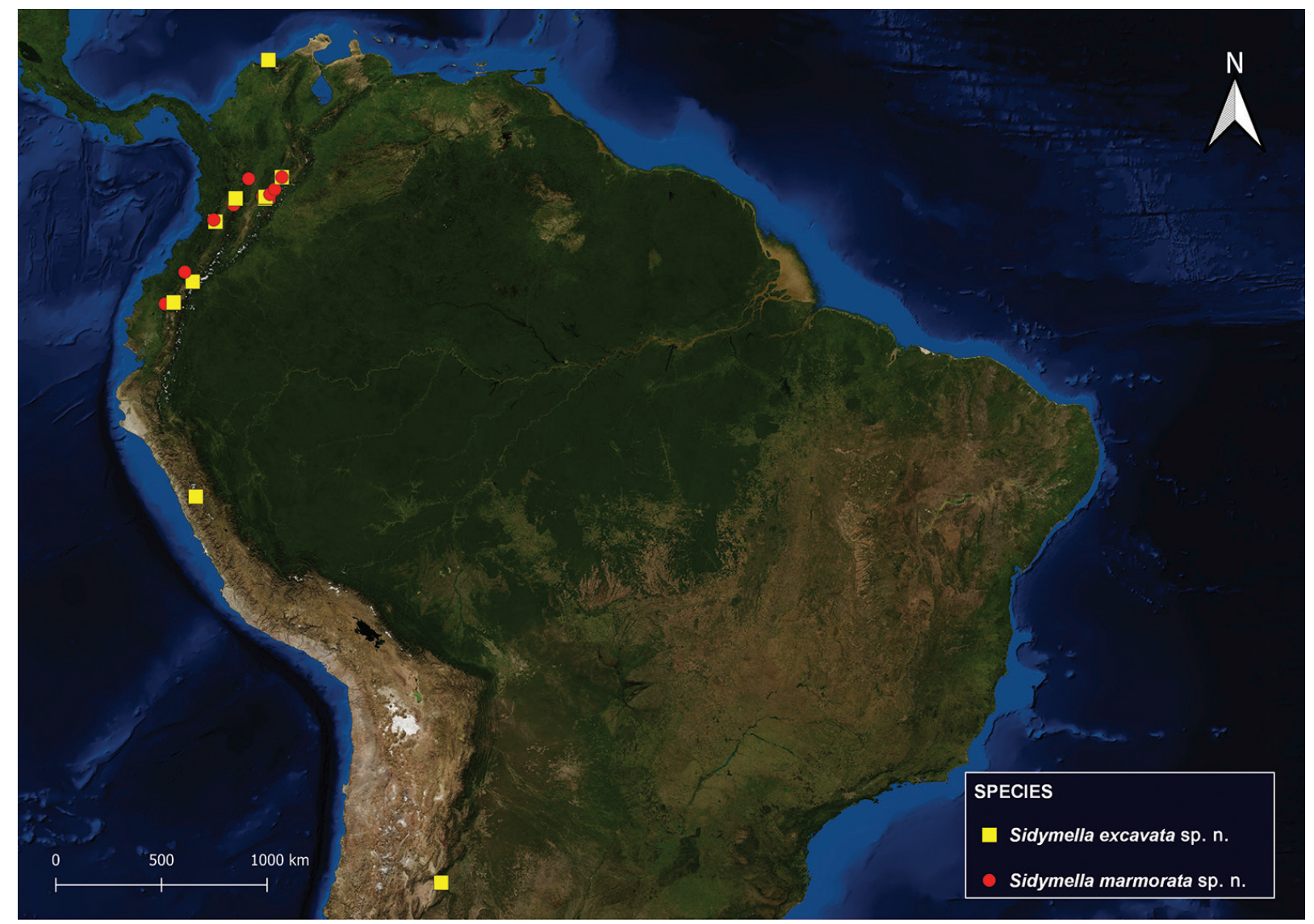

Figure 15. Distribution records of Sidymella excavata sp. nov. and Sidymella marmorata sp. nov.

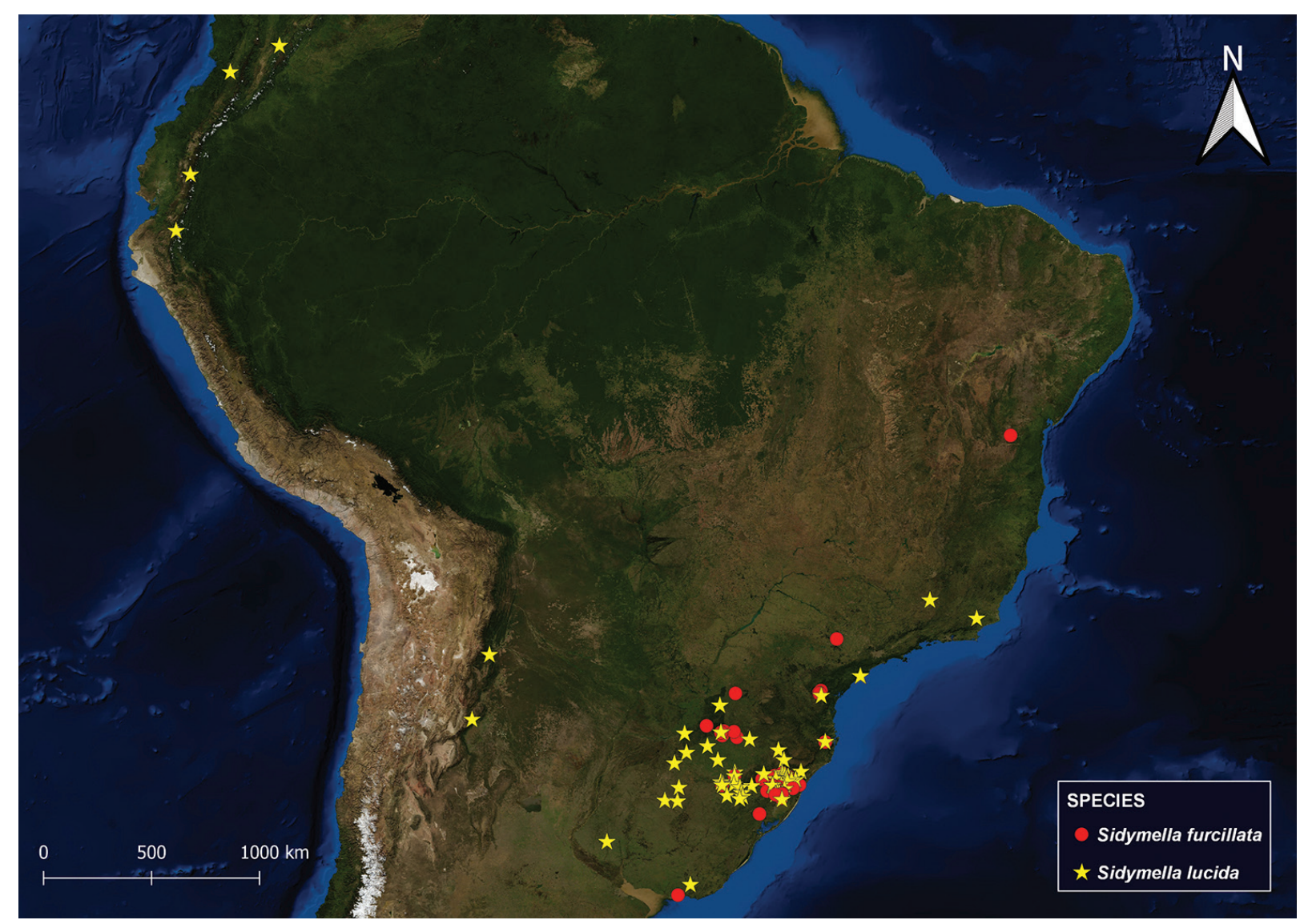

Figure 16. Distribution records of Sidymella furcillata and Sidymella lucida. 


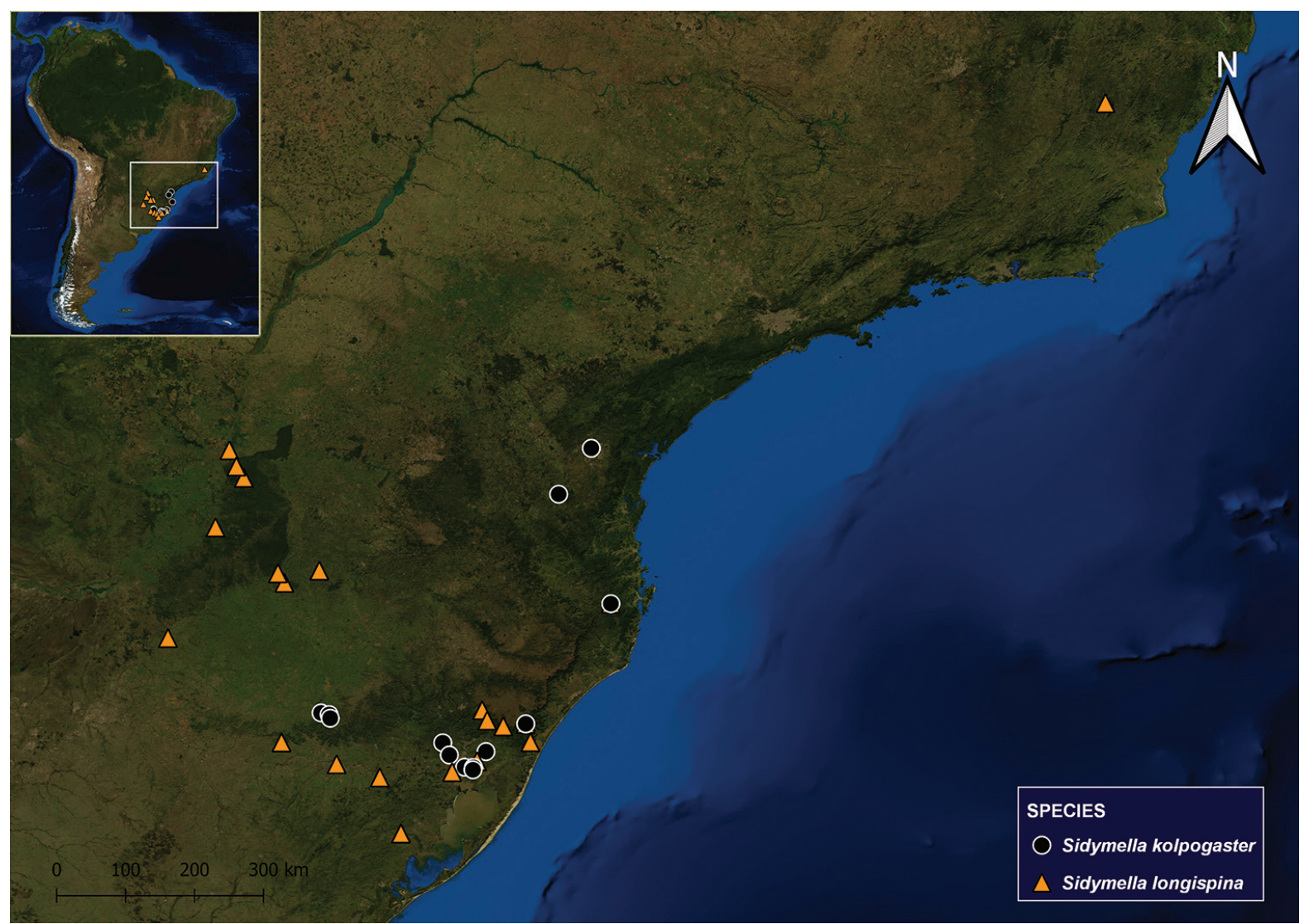

Figure 17. Distribution records of Sidymella kolpogaster and Sidymella longispina.

\section{Additional taxonomic changes}

Sidymella nigripes Mello-Leitão, 1947: 276 (ð). Lise 1973: 16, figs 34-38 (ㅇ).

Note. The holotype female was collected in Curitiba by Padre Moure in April 1942 and deposited in the Instituto de Proteção do Patrimônio Natural da Secretaria da Agricultura do Estado do Paraná under the number 2497. However, this institution has closed and its arthropod collection was transferred to unknown institutions. The repository of this species is unknown, even after we searched for it in all major Brazilian institutions with arachnological collections, and we assume that the holotype of $S$. nigripes is lost. Therefore, we propose that the species should be considered a species inquirenda.

Sidymella obscura Mello-Leitão, 1929: 65. Holotype juvenile collected in Serro da Caraça, Minas Gerais, Brazil (MNHN 8263, examined). Nomen dubium.

Sidymella parallela Mello-Leitão, 1929: 64. Holotype juvenile collected in Salobro, Bahia, Brazil (MNHN 31114, examined). Nomen dubium.

Sidymella spinifera Mello-Leitão, 1929: 66. Lise 1973: 21, figs 47-53. Syntypes, 1 immature $\widehat{\partial}$ and 1 immature $q$ collected in Serro da Caraça, Minas Gerais, Brazil (MNHN 8202, examined).
Note. In the original work, Mello-Leitão (1929) mentioned both specimens as "type", but they are labelled as syntypes. Both specimens are juveniles that cannot be diagnostic of any species. Nomen dubium.

\section{Acknowledgments}

We thank all curators who provided the material or access to the collections to examine the specimens listed above. We are also thankful to the staff of the Centro de microscopia e microanálises (CEMM) of the Pontifícia Universidade Católica do Rio Grande do Sul (PUCRS) for technical support, to Diego Galarraga Sugoniaev and Damián Hagopián for the images of live specimens, and to Dr Arthur Anker for the examination and photos of the holotypes of S. obscura, S. parallela, and S. spinifera. We thank the referees and Steven Chu for comments and suggestions that helped to improve this manuscript. This study was financed in part by the Coordenação de Aperfeiçoamento de Pessoal de Nivel Superior, Brasil (CAPES) (Finance Code 001).

\section{References}

Benjamin SP, Dimitrov D, Gillespie RG, Hormiga G (2008) Family ties: molecular phylogeny of crab spiders (Araneae: Thomisidae). Cladistics 24: 708-722. https://doi.org/10.1111/j.1096-0031.2008.00202.x 
Benjamin SP (2011) Phylogenetics and comparative morphology of crabs piders (Araneae: Dionycha, Thomisidae). Zootaxa 3080: 1-108. https://doi.org/10.11646/zootaxa.3080.1.1

Bonaldo AB, Lise AA (2001) A review of the Neotropical spider genus Stephanopoides (Araneae, Thomisidae, Stephanopinae). Biociências 9: 63-80.

Keyserling E (1880) Die Spinnen Amerikas, I. Laterigradae. Bauer \& Raspe (E. Küster), Nürnberg, 283 pp. https://doi.org/10.5962/bhl. title. 64832

Lise AA (1973) Contribuição ao conhecimento do gênero Sidyma no Brasil, com descrição de uma nova espécie (Araneae-Thomisidae). Iheringia 43: 3-47.

Lise AA (1981) Tomisídeos Neotropicais V: Revisão do gênero Onocolus Simon, 1895 (Araneae, Thomisidae, Stephanopinae). Iheringia 57: 3-97.

Machado M, Teixeira RA, Lise AA (2015) Taxonomic notes on the crab spider genus Tobias Simon, 1895 (Araneae,Thomisidae, Stephanopinae). Zootaxa 4034(3): 565-576. https://doi.org/10.11646/zootaxa.4034.3.8

Machado M, Teixeira RA, Lise, AA (2017) Cladistic analysis supports the monophyly of the Neotropical crab spider genus Epicadus and its senior synonymy over Tobias (Araneae: Thomisidae). Invertebrate Systematics 31: 442-455. https://doi.org/10.1071/IS16074

Mello-Leitão CF (1929) 'Aphantochilidas e Thomisidas do Brasil.' Archivos do Museu Nacional do Rio de Janeiro: Rio de Janeiro, Brazil, 359 pp.
Prado AW, Baptista RL, Machado M (2018) Taxonomic review of Epicadinus Simon, 1895 (Araneae: Thomisidae). Zootaxa 4459: 201234. https://doi.org/10.11646/zootaxa.4459.2.1

Ramírez MJ (2014) The morphology and phylogeny of dionychan spiders (Araneae: Araneomorphae). Bulletin of the American Museum of Natural History 390: 1-374. https://doi.org/10.1206/821.1

Silva-Moreira T, Machado M (2016) Taxonomic revision of the crab spider genus Epicadus Simon, 1895 (Arachnida: Araneae: Thomisidae) with notes on related genera of Stephanopinae Simon, 1895. Zootaxa 4147(3): 281-310. https://doi.org/10.11646/zootaxa.4147.3.4

Simon E (1895) Histoire naturelle dês araignées. Librairie Encyclopédique de Roret, Paris, 1084 pp. https://doi.org/10.5962/bhl.title.47654

Strand E (1942) Miscellanea nomenclatorica zoologica et palaeontologica. X. Folia Zoologica et Hydrobiologica 11: 386-402.

Wheeler WC, Coddington JA, Crowley LM, Dimitrov D, Goloboff PA, Griswold CE, Hormiga G, Prendini L, Ramírez MJ, Sierwald P, Almeida-Silva L, Alvarez-Padilla F, Arnedo MA, Benavides LR, Benjamin SP, Bond JE, Grismado CJ, Hasanf E, Hedin M, Izquierdo MA, Labarque FM, Ledford J, Lopardo L, Maddison WP, Miller JA, Piacentini LN, Platnick NI, Polotow D, Silva-Dávila D, Scharff N, Szúts T, Ubick D, Vink CJ, Wood HM, Zhang J (2017) The spider tree of life: phylogeny of Araneae based on target-gene analyses from an extensive taxon sampling. Cladistics 33: 574-616. https://doi.org/10.1111/cla.12182

World Spider Catalog (2019) World Spider Catalog. Natural History Museum Bern. Version 20.0. http://wsc.nmbe.ch [accessed March 2019] 